This report was prepared as an account of work sponsored by an agency of the United States Government. Nieither the United States Government nor any agency thereor, nor any of their empioyes, make any warranty, express or implied, or issumes any legal liability or responsibitity for the aceuracy, completeness, or usefulness of any information, apparatus, product, or process disclosed, of represents that its use wauld not inftinge prisately owned rights. Reference herein to any specifis commercial product, process, or serviee by trade name, trademath. monufacturet, or atherwise does not necessarily constitute or imply its endorsement, recomnendation, or favoring by the Uniled States Govemment or any agency thereof. The views and opinions of authors cxpressed herein do not necessarily state of reflect those of the United States Government or any agency thereof.

\title{
A nonvariational code for calculating three-dimensional MHD equilibria
}

\author{
H. S. Greenside; A. H. Reiman, and A. Salast \\ Plasma Physics Laboratory, Princeton University \\ Princeton, New Jersey 08544-0451
}

\begin{abstract}
Details are presented of the PIES code, which uses a nonvariational algorithm for calculating fully threedimensional MHD equilibria. The MHD equilibrium equations are directly iterated in special coordinates to find selfconsistent currents and magnetic fields for given pressure and current profiles and for a given outermost magnetic surface. Three important advantages of this approach over prevjous methods are the ease with which net current profiles can be imposed, the explicit treatment of resonances, and the ability to handle magnetic islands and stochastic field lines. The convergence properties of the code are studied for several axisymmetric and nonaxisymmetric fnite- $\beta$ equilibria that have magnetic surfaces.
\end{abstract}

-Permanent address: Department of Computer Science, Duke University, Durham, N. J. 27706 .

Permanent address: Departmento de Fisica, E. T. S. de Ingenieros Industriales, Unjversidad Politécnica de Madrid, José Gutiérrez Abascal, 2, 28006 Madrid, Spain. 


\section{INTRODUCTION}

Numerical calculations of toroidal magnetohydrodynamic (MHD) equilibria play an important role in the design of tokamaks and stellarators and in the interpreiation of experimental data. These calculations are difficult for two reasons. First, laboratory plasmas are intrinsically three dimensional. Tokamaks, for example, use discrete toroidal field coils that add nonaxisymmetric perturbations (ripple) to a nominally axisymmetric device. Tokamak piasmas are also often unstable to symmetry-breaking tearing modes. Stellarators use nonaxisymetric coils to obtain a rotational transform so that even the stellarator vacuum field is three dimensional. A consequence of this three-dimensional structure is that magnetic surfaces can not exist throughout the volume of the plasma. ${ }^{3}$ Numerical algorithms are therefore needed that can calculate MHD equilibria without assuming the existence of magnetic surfaces.

A second difficulty in calculating MHD equilibria is the high-dimensional parameter space that characterizes toroidal fusion devices (e.g., aspect ratio, coil winding law, number of periods, current profile, anc pressure profile). Many equilibria must be calculated to map out and to understand this highdimensional domain. Numerical algorithms that converge rapidly and efficiently are therefore very important for studying equilibria. Also important are algorithms that converge robustly when the magnetic surfaces of $\mathbf{B}$ are strongly distorted, e.g., because of large shifts of the magnetic axis at high $\beta$. Even at lower $\beta$, experimentalists may choose to use highly shaped plasmas to avoid certain jdeal MHD instabilities. ${ }^{2}$

In this paper, we present details of a new computer code that shows significant promise in overcoming these difficulties. We have named this code PIES, an acronym for the Princeton Iterative Equilibrium Solver. The PIES code solves the MHD equilibrium equations

$$
\begin{aligned}
\mathbf{J} \times \mathbf{B} & =\nabla p \\
\nabla \times \mathbf{B} & =\mathbf{J} \\
\nabla \cdot \mathbf{B} & =0
\end{aligned}
$$

in a general toroidal domain, where $\mathbf{J}$ is the current density, $\mathbf{B}$ is the magnetic field, and $\nabla p$ is the pressure gradiunt. As we show below, our code converges rapidly and efficiently, and should be useful for exploring the high-

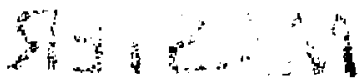


dimensional parameter space of fusion confinement devices. 1 here is no assumption that magnetic surfaces exist, so magnetic islands and stochastic field lines can be treated (although their treatment requires some additional numerical machinery not discussed in this paper).

The algorithm is simple to state, although subtle to implement. ${ }^{3,5,6}$ The MHD equations, Eqs. (1)-(3), are iterated, one after another, to find a magnetic field $\mathbf{B}$ and a current density $\mathbf{J}$ that are consistent with given pressure and current profiles and with a given outermost magnetic surface. Given an initial guess for $\mathbf{B}, \mathrm{Eq}$. (1) is solved for the current density $\mathbf{J}$. This requires evalution of various quantities along the fieid lines of $B$, which explains why the code is sensitive to the magnetic field line topology. Given the new current density $\mathrm{J}, \mathrm{Eq}$. (2) is used to solve for a new $\mathbf{B}$ that is divergence-free. This process is repeated until acceptable convergence is attained. Since this algorithm obtains solutions without minimizing a functional, we call this a nonvariational code.

Nonvariational three-dimensional equilibrium codes have not been studied previously, so that the algorithm and its convergence properties for equilibria with magnetic surfaces are of immediate interest. In this paper, we explain the details of the algorithm and demonstrate convergence for representative axisymme'ric and nonaxisymmetric equilibria that have magnetic surfaces. We also examine how the convergence rates depend on both physical and numerical parameters. In the future, we hope to apply this code to study toroidal ripple and tearing modes in tokamaks and equilibrium $\beta$ limits in stellarators.

The PIES code required two significant numerical advances that are described in detail elsewhere. First, we derived an algorithm that accurately solves magnetic differential equations by transforming operators and fields into magnetic coordinates (in which the field lines of $B$ appear straight) ${ }^{6}$ This changes a differential equation into an algebraic equation that is easily solved. This transformation requires evaluating a given scalar field along magnetic field lines, Fourier decomposing the resulting "time series" along the field lines, and then identifying the one-dimensional Fourier coefficients with the two-dimensional Fourier expansion of this scalar in the magnetic surfaces defined by the field lines. Although algorithms for this have been described in the literature, ${ }^{8,9}$ they were not satisfactory for an equilibrium code. The identification of the two-dimensional Fourier coefficients on a mag- 
netic surface with the one-dimensional Fourier coefficients along a field line defining that magnetic surface was not automated. Also, specification of the required accuracy of Fourier coefficients was not possible.

Our second numerical advance was the implementation of a second-order accurate three-dimensional Poisson solver with Neumann boundary conditions that uses numerically defined coordinates. This allows the solution of Eqs. (2) and (3) in magnetic coordinates or in any other useful coordinate system (such as optimized coordinates ${ }^{10}$ that can reduce the computational effort). This solver turned out to be the most delicate part of the algorithm. The overall convergence rate and numerical stability of the PIES code depended sensitively on details of the radial discretization of the Poisson solver. Development of a robust code required a systematic study of different discretizations.

In what follows, we will assume as given the numerical methods and computer codes for solving magnetic differential equations and for solving Poisson equations with Neumann houndary conditions. This will allow us to concertrate on the details and subtleties of implementing the nonvariatjonal algorithm of the PIES code. Where needed, details can be obtained from Refs. 6 and a forthcoming publication on the Poisson solver.

The rest of the paper is arranged as follows: In Section II, we give an overview of the PIES code. The succeeding sections expand Section II, giving more details about the physics and about the numerical methods. In Section III, we explain the coordinate systems that are used and how the metric elements are calculated. In Section IV, we discuss the numerical implementation of the nonvariational method. In Section V, we examine axisymmetric and three-dimensional MHD equilibria and analyze the convergence properties of the algorithm with respect to various pararieters. In Section VI, we give a summary and conclusions.

Two appendices contain discussions that are too detailed or that are of lesser importance to be included in the main text. We give a simple analytic example to illustrate the basic algorithm, and we discuss how the Fourier cseffi ients of the magnetic field from the previous iteration can be expressed in the present magnetic coordinates.

An earlier summary of work on this code was given in Ref. 3. A closely related and independent effort, using quite different numerical methods, has been described by Kjsslinger and Wöbig. ${ }^{11}$ Other nonvariational algorithms 
have been proposed ${ }^{12,13}$ but have not yet bees: implemented and tested. Researchers have also independently pursued an algorithm similar to the PIES code in nontoroidal geometries, solving the Euler equations for inviscid flow in turning channels. ${ }^{14}$ However, many of the subtletjes of the MHD equations in toroidal domains (large shifts of the magnetic axis, resonances on rational surfaces, and island formation) do not occur in this context.

\section{Overview}

In this section, we give an overview of the physics and numerical analysis that entered into the design of the PIES code. Later sections expand this discussion in detai].

Our main motivation for writing the PIES code was our dissatisfaction with present algorithms for studying magnetic island formation and the onset of stochasticity. Time-dependent three-dimensional codes ${ }^{15}$ require long expensive integrations over fast time scales and are not practical for systematic studies in a high-dimensional parameter space. Previous three-dimensional equilibrium codes, ${ }^{16-18}$ although they avoid the integrations over fast time scales, are not useful for investigating the crucial question of the appearance and interaction of magnetic islands since they build in the assumption of magnetic surfaces.

These equilibrium codes are also variational in that they find an equilibrium by minimizing the plasma energy under certain constraints. It is questionable whether a variational approach is best for investigating large$\beta$ equilibria and magnetic island formation. Significant topological changes in the magnetic field can arise from tiny changes in the energy functional. Furthermore, variational principles-as is well known-are best at estimating the values of extrema and not at estimating the field's that achieve the extrema. This suggests that a nonvariational iterative method may converge more rapidly and represent the magnetic topology more accurately. Analytical calculations support this view. ${ }^{19}$

Nonvariational methods for solving the MHD equations were actually among the first to be suggested, ${ }^{20,21}$ and closely related algorithms were successfully implemented in Grad-Shafranov solvers for axisymmetric equilibria. ${ }^{22}$ Interest in nonvariational methods for three-dimensional equilibria was stimulated by recent papers of Boozer, who explored the advantages of a 
particular nonvariational algorithm in magnetic coordinates. ${ }^{5,8,23}$ He showed anajytically that the Shafranov shift, an $m=1$ translation of the magnetic axis as $\beta$ increases, is physically and economically treated, and that one can easily impose net current profiles (which is awkward for variational codes). An analytic calculation also demonstrated the capability of the method to determine island widths and equilibrium $\beta$ limits for a heliac device ${ }^{19}$ This latter calculation suggested that a nonvariational formalism was a physically useful starting point for studying complicated MHD equilibria. These studies left unanswered, however, the convergence properties of such algorithms.

Our nonvariational iteration of the MHD equations is basically as follows. First, the current density $J$ is obtained in terms of certain profiles and the initial magnetic field. Given a pressure profile $p(\rho)$, a net poloidal current profile $g(\rho)$, a net toroidal current profile $J(\rho)$, and the initial magnetic field $B$, Eqs. (1) and (2) yield a magnetic differential equation for a single-valued stream function $\nu$, from which the current density $\mathbf{J}$ can be calculated [see Eq. (38) below]:

$$
(\boldsymbol{B} \text { - } \nabla) \nu=p^{\prime}+\frac{\mathfrak{g}^{\prime}- \pm I^{\prime}}{\mathcal{J}^{p}}
$$

$t(\rho)$ is the rotational transform (winding number) of the magnetic field line defining the magnetic surface labeled by a radial variable $\rho, \mathcal{J}^{\rho}$ is the Jacobian between laboratory and magnetic coordinates [see Eq. (29) below], and ' denotes differentiation with respect to $p$. Equation (4) yields both the diamagnetic current density $\left(J_{\perp}=\mathbf{B} \times \nabla p / B^{2}\right)$ and the Pfirsch-Schluter current densities $\left(\mathbf{J}_{\|}=\mathbf{J}-\mathbf{J}_{\perp}\right)$ upon solution.

This hyperbolic equation is the physically challenging part of iterating the MHD equations; it is at this point that the treatment of magnetic islands and stochastic regions enters. In this paper, we deal only with feld lines lying in good magnetic surfaces. For such field lines, Eq. (4) can be solved by transforming the right side and $\mathbf{B}$ into magnetic coordinates. (This transformation is done numerically using the algorithm of Ref. 6.) This diagonalizes the operator B $\cdot \nabla$, giving an algebraic equation that is trivially solved for the Fourier coefficients (in magnetic coordinates) of the unknown $\nu$.

Once $\psi$ and hence $J$ are known, we can next solve Ampère's law, Eq. (2), for a new magnetic field $\mathbf{B}$ that is also divergence free Eq. (3). This involves solving an elliptic equation (a Poisson equation). Discretizing the elliptic equation leads to a block tridiagonal matrix that is readily solved 
given existing software. ${ }^{24}$ Ampère's law is the numerically difficult part of the code because overall convergence depends on subtle details of how the Poisson equation is discretized, e.g., whether a conservative discretization is chosen, or whether Fourier coefficients are defined on half-integer radial meshes as opposed to integer radial meshes. This numerical sensitivity arises partly because the coordinate system is centered on a magnetic axis which varies from iteration to iteration, and also because some quantities (metric elements, vector components) can become singular near the magnetic axis.

From a numerical point of view, the above algorithm has the advantage of formulating the solution of the MHD equations as the solution of two linear equations, a hyperbolic magaetic differential equation for the current stream function, and an elliptic equation for the new magnetic field from Ampère's law. Numerical methods that are especially adapted to each type of equation can be used. The nonlinearities of the MFD equations enter through integrations along magnetic field lines and through the change in coordinates at each iteration, as described in Section III below.

The numerical representation of fields used in the PIES code was dictated primarily by the computational complexity of solving the Puisson equation derived from Ampère's law. This complexiry grows rapidly as a power of the angular resolution, becoming asymptoticaliy larger than the effort needed to follow fields lines and to solve a magnetic differential equation, which grows approximately linearly with increasing poloidal resolution.

In a toroidal domain, Fourier representations in the poloidal and toroidal variables for all fields are a natural choice, and they are known to reduce the computational effort of solving the MHD equations compared to using a real space mesh. ${ }^{25}$ Substituting finite Fourjer representations into Ampère's law leads to coupled second-order ordinary differential equations for the radial dependence of the Fourier coefficients of the unknown magnetic field. For general nonseparable coordinate systems iwhich are needed to obtain efficient representations of distorted magnetic surfaces), the Fourier coefficients of the metric elements couple to the Fourier coefficients of the solution to give a block matrix on each magnetic surface. Second-order accurate finite-difference approximations to radial derivatives couple nearest neighbor surfaces to a given surface, giving a block tridiagonal matrix. This matrix can be solved readily and efficiently with existing software. ${ }^{24}$

More global radial basis functions couple many magnetic surnaces together 
and lead to much larger matrices to invert. Chebyshev polynomials in particular would lead to dense block matrices in the solution of Anpères law, whose storage and solution would pose serious problems, even on today's supercomputers. Furthermore, iterative methods would be needed to invert such large matrices. We have preferred to use finite differences and direct (noniterative) methods to avoid inner iterations, which would complicate convergence studies of the algorithm. We note, however, that higher-order basis functions may be useful in the numerical algorithms that transform fields into magnetic coordincies. ${ }^{6}$ This algorithm requires interpolation of $B$ throughout the domain of interest when following different field lines, which would be more accurate if inore global representations were used.

In summary, we solve the MHD equations iteratively and nonvariationally by calculating first the currents driven by a given magnetic field with prescribed pressure and current profiles. Then we calculate the magnetic field generated by these currents. Essential to the success of the code are algorithms for efficiently solving a magnetic differential equation ${ }^{6}$ and Ampère's law. The magnetic differential equation is solved foi field lines lying in magnetic surfaces by a numerical transformation to magnetic coordinates in which the field lines are straight. Ampère's law is reduced to a single threedimensional Poisson equation with Neumann boundary conditions, which is solved by Gaussian elimination, using a previously witten memory-efficient software package. ${ }^{24}$

\section{Coordinates and Metric Elements}

In this section, we discuss the coordinate systems used in the PIES code, how fields are represented numerically, and how the metric elements are calculated. Our approach is to use a generalization of flux coordinates, for which constant radial surfaces coincide with magnetic surfaces where they exist, and which interpolate through magnetic islands and stochastic regions. All fields are expressed as Fourier series in two angular variables, a poloidal angle $\theta$, and a toroidal angle $\phi$. The Fourier coefficients will be known on a discrete uniform radial mesh labeled by $\rho$ (we will also sometirnes use another radial variable $\psi$ ).

An advantage of using flux, rather than laboratory, coordinates is that most of the rapid variation of fields occurs in the radial direction. Fewer 
harmonic modes are needed to represent variation in the toroidal or poloidal directions, reducing substantially the computational effort of the code.

\section{A. Coordinates}

The coordinates $(\rho, \theta, \phi)$ are determined by the field lines of $\mathbf{B}$ at each iteration of the PIES cade. ${ }^{8}$ For equilibria with magnetic surfaces, they are magnetic coordinates, with constant $\rho$ surfaces coinciding with magnetic surfaces. Both $\rho$ and $\theta$ change with $\mathbf{B}$, while the toroidal angle $\phi$ remains the same. Fixing $\phi$ to be the usual cylindrical $\phi$ removes the ambiguity in $\theta$ that exists in magnetic coordinates. It is also a convenient choice for plotting magnetic surfaces in laboratory coordinates.

The toroidal domain in which the MHD equations will be: solved is defined in these coordinates by:

$$
\begin{aligned}
& 0 \leq \rho \leq 1 ; \\
& 0 \leq \theta \leq 2 \pi ; \\
& 0 \leq \phi \leq 2 \pi .
\end{aligned}
$$

This domain includes any geometry that is periodic in the two angles $\theta$ and $\phi$, e.g., a torus, a periodic cylinder, and a periodic helix whose cross section is a smooth deformation of a circle. The closed loop defined by $p=0$ is chosen to coincide with a magnetic axis of $B$, while the surface $\rho=1$ will coincide with a given outermost magnetic surface, corresponding to a perfectly conducting shell enclosing the plasma. An algorithm for following fields lines of $\mathbf{B}$ and obtaining coordinates $(\rho, \theta, \phi)$ that satisfy these constraints was given in Ref. 6.

This domain can also be considered to define one period of a device that is not axisymmetic. In that case, we can replace $\phi$ by $N \phi$ and $\partial_{\phi}$ by $N \partial_{\phi}$ to treat the case of a device with $N$ periods. In what follows, we shall assume a single-period machine $(N=1)$.

For simplicity, we also assume that the domain is symmetric under the so-called stellarator symmetry:

$$
\theta \rightarrow-\theta ; \quad \phi \rightarrow-\phi .
$$

Most stellarator vacuum fields have this symmetry, and the generalization to devices lacking this symmetry is straightforward. With this assumption, 
scalar fields will have Fourier series that have even or odd parity under this transformation, of the form

$$
f(\rho, \theta, \phi)=\sum_{m=0}^{M} \sum_{n=-N}^{N} f_{m, n}(\rho) \cos (n \phi-m \theta),
$$

for even parity, and of the form

$$
f(\rho, \theta, \phi)=\sum_{m=0}^{M} \sum_{n=-N}^{N} f_{m, r}(\rho) \sin (n \phi-m \theta),
$$

for odd parity. Here $M$ and $N$ are nonnegative integers that determine the modal resolution in the poloidal and toroidal directions respectively. Table I summarizes the parities of various fields used in the code.

The Fourier coefficients $f_{m, n}(\rho)$ are assumed to be known on either of two uniform radial meshes, an integer mesh commensurate with $p=0$ and with $p=1$ :

$$
\rho_{l}=l d \rho, \quad l=0, \ldots, L ;
$$

or a half-integer mesh that is shifted half a radial mesh spacing with respect to the integer mosh,

$$
p_{l+1 / 2}=\{l+1 / 2\} d p, \quad l=0, \ldots, L-1 .
$$

The radial mesh spacing is $d \rho=1 / L$, where $L$ is some positive irtriger determining the radial resolution. We will use superscript $l$ to denote the value of a Fourier coefficient at the lth mesh point: $a_{m, n}^{l}=a_{m, n}(l d \rho)$, with a similar notation for the half-integer mesh.

Each scalar field therefore has $(L+1)(\mathcal{M}+1)(2 N+1)$ degrees of freedom, and is stored as a Fortran 77 real array of the form $f(0: M,-N: N, 0: L)$. In the aisence of the stellarator symmetry, $m$ would vary from $-M$ to $M$, and twice as many degrees of freedom would be required for given $M$. We note that the representation:, Eqs. (7) and (8), have redundant coefficients since the terms

$$
f_{0, n}(\rho)= \pm f_{0,-n}(\rho),
$$

are identical for $n>0$. This redundancy is intentional and serves two useful purposes. First, it allows the parity of each array to be tested, which is useful for verifying the correctness of a long calculatio: that produced that array. 
Second, it allows vectorization of Fortran do loops using the natural bounds, do $m=0, M$, do $n=-N, N$, an: $\mathbf{I}$ do $I=0$, L.

The magnetic ccordinates are related at each iteration to a laboratory coordinate system. Instead of using Cartesias coordinates $(X, Y, Z)$, at is more computationally useful to use a rotating laboratriy coordinate system $(x, y, z)$, which is shown in Fig. 1; these coordinates riquire fewer Fourier coeff.cients when expressed in magnetic coordinates. ${ }^{36}$ For finite aspec: ratio donains, $z=-R \phi$, where $R$ is the major radius and $\phi$ is the toroidal angle of a cylindrical coodinate system, while for infinite aspect ratio domains, $z$ becomes the usual constant Carterian coordinate $Z$. An arbitrary vector car then be written in the following form:

$$
\mathbf{X}(\rho, \theta, \phi)=x(\rho, \theta, \phi) \hat{\mathbf{x}}+y(\rho, \theta, \phi) \hat{\mathbf{y}}
$$

where the unit vectors are given in Cartesian coordinates by:

$$
\begin{aligned}
& \hat{\mathbf{x}}=-\cos (\phi) \hat{X}+\sin (\phi) \hat{Y}, \\
& \hat{\mathbf{y}}=\hat{Z}, \\
& \hat{\mathbf{z}}=\partial_{\phi} \hat{\mathbf{x}}=\hat{\mathbf{x}} \times \hat{\mathbf{y}}=\sin (\phi) \hat{\mathbf{X}}+\cos (\phi) \hat{\mathbf{Y}} .
\end{aligned}
$$

These definitions are appropriate for a fi.ite aspect ratio torus. For infinite aspect ratio cylinders, the coordinates are Cartesian:

$$
\begin{gathered}
\mathbf{X}(\rho, \theta, \phi)=x(\rho, \theta, \phi) \hat{\mathbf{x}}+y(\rho, \theta, \phi) \hat{\mathbf{y}}+R \phi \hat{\mathbf{z}} \\
\hat{\mathbf{x}}=\hat{Y}_{;} \quad \hat{\mathbf{y}}=\dot{Z}_{;} \quad \hat{\mathbf{z}}=\hat{\mathbf{x}} \times \hat{\mathbf{y}}=\hat{\mathbf{X}},
\end{gathered}
$$

where $R$ is now the effective major radius of the cylinder ( $2 \pi R$ is the length of the magnetic axis).

At the beginning of each iteration of the PIES code, the coordinates $x$ and $y$ are expressed in Fourier series of the forms given by Eqs. (7) and (8), respectively. This is achieved ${ }^{6}$ by evaluating $x$ and $y$ along fields lines of the magnetic field B. Once these Fourier series are known, we can calculate the metric elements of the magnetic coordinate system defined implicitly by $B$. This requires evaluating numerical derivatives of various Fourier series and convoluting the series in pairs to avoid aliasing. We first discuss how derivatives are calculated. 


\section{B. Derivatives of Fourier Coefficients}

Derivatives first arise in evaluating the Fourier coefficients of the covariant coordinate vectors

$$
\begin{aligned}
& \mathbf{e}_{\rho}=\mathrm{X}_{, \rho}=x_{, \rho} \hat{\mathbf{x}}+y_{, \rho} \hat{\mathbf{y}}, \\
& \mathrm{e}_{\theta}=\mathbf{X}_{, \theta}=x_{, \theta} \hat{\mathbf{x}}+y_{, \theta} \hat{\mathbf{y}}, \\
& \mathbf{e}_{\phi}=\mathbf{X}_{, \phi}= \begin{cases}x_{, \phi} \hat{\mathbf{x}}-y_{, \phi} \hat{\mathbf{y}}+x \hat{\mathbf{z}} & \text { finite aspect ratio, } \\
x_{, \phi} \dot{\mathbf{x}}+y_{, \phi} \hat{\mathbf{y}}+R \hat{\mathbf{z}} & \text { infinite aspect ratio, }\end{cases}
\end{aligned}
$$

which are needed in constructing the covariant metric elements $g_{i j}=\mathbf{e}_{i} \cdot \mathbf{e}_{j}$. In Eqs. (18)-(20), we use a comma notation to indicate partial derivatives with respect to a variable, e.g., $\mathbf{X}_{, \rho}=\partial \mathbf{X} / \partial \rho$, with similar expressions for $\theta$ and $\phi$ derivatives.

The angular derivatives are obtained in the usual way, multiplying Fourier coefficients by mode numbers. For example, for $x$ :

$$
\begin{aligned}
& x_{, \theta}=\sum\left[m x_{m, n}(\rho)\right] \sin (n \phi-m \theta) ; \\
& x_{, \phi}=\sum\left[-n x_{m, n}(\rho)\right] \sin (n \phi-m \theta),
\end{aligned}
$$

with similar expressions for the angular derivatives of $y$. The angular derivatives change the parjty of the array under the stellarator symmetry.

The radial derivatives of Fourier coefficients require more careful consideration. Straightforward finite difference approximations to the radial derivatives of Fourjer coefficients $f_{m, n}(\rho)$ give large relative errors near the magnetic axis for large poloidal mode numbers $m$, of $O(1)$ instead of $O\left(d p^{2}\right)$. We found it necessary to modify the finite difference expressions to avoid this problem.

The reason for the large relative error in the radial derivative is that second-order accurate finite differences are reasonable only when $f_{m, n}(\rho)$ is locally well approximated by a quadratic curve. This is generally not the case in polar-like coordinates near the polar origin. Fourier coefficients vary near the magnetic axis as a power of $\rho$ times a power series in $\rho^{2}$ :

$$
f_{m, n}(\rho) \simeq \rho^{e_{s}(m)}\left(f_{m, n}^{0}+f_{m, n}^{2} \rho^{2}+\cdots\right), \quad \rho \ll 1,
$$

where $f_{m, n}^{0}, f_{m, n}^{2}, \ldots$, are constants and where $e_{f}(m)$ is an integer function that depends on the scalar $f$. For analytic functions $\mathcal{A}, e_{\mathcal{A}}(m)=m$. [Analytic functions $\mathcal{A}(\rho, \theta, \phi)=\mathcal{A}(\xi, \eta, \phi)$ have bounded Taylor series near the 
magnetic axis when expressed in the Cartesian-like variables $\xi=\rho \cos (\theta)$ and $\eta=\rho \sin (\theta)$.] For metric elements and other more complicated combjnations of analytic functions, $e_{f}(m)$ is approximately $m$ for large $m$. Table I summarizes the leading radial powers, $e_{f}$, for various fields $f$ that are used in the PIES code.

Equation (23) suggests that the large relative error in radial derivatives near $\rho=0$ can be reduced by introducing radial factor $3(f)_{m}(\rho)$ whose derivative can be taken analytically, and such that $f_{m, n}(\rho) / R(f)_{m}(\rho)$ is locally quadratic. One reasonable choice would be

$$
R(f)_{m}(\rho)=\rho^{\min \left(e_{f}(m), m i\right)} .
$$

The radial factor depends on both the mode number and particular function $f$. The integer $\bar{m}$, which satisfies $1 \leq \bar{m} \leq m$, prevents $R(f)_{m}(\rho)$ from becoming too small for large $m$ and small $\rho$. As we will see in Section IV, this cutoff is necessary when solving Ampère's law. The discretized equations of Ampère's law become ill-conditioned near the magnetic axis if too large radial powers are pulled out. In practice, $\bar{m} \simeq 2$ gives satisfactory results, balancing the need to obtain increased accuracy near the magnetic axis with the need to avoid jll-conditioning with tiny numbers.

We thus define the radial derivative at the mesh value $\rho_{l}=l d \rho$ to be

$$
\left(\frac{\partial}{\partial \rho} f\right)_{m, n}^{l}=\left(\frac{\partial}{\partial \rho} R(f)_{m}\right)^{l} \frac{f_{m, n}^{l}}{R(f)_{m}^{l}}+R(f)_{m}^{l} \delta_{p}\left(\frac{f_{m, n}}{R(f)_{m}}\right)^{l} .
$$

Here $\delta_{\rho}$ represents the appropriate second-order accurate finite-difference operato: On the integer mesh, it takes the form

$$
\left(\delta_{p} a_{m, n}\right)^{l}= \begin{cases}2\left(a_{m, n}^{2}-a_{m, n}^{1}\right) /(3 d \rho) & l=1, \\ \left(a_{m, n}^{l+1}-a_{m, n}^{l-1}\right) /(2 d \rho) & 1<l<L, \\ \left(3 a_{m, n}^{l}-4 a_{m, n}^{l-1}+a_{m, n}^{l-2}\right) /(2 d \rho) & l=L,\end{cases}
$$

with a similar expression for the derivative on the half-integer mesh in terms of the values on the integer mesh. The second-order accurate two-point discretization next to the magnetic axis $(l=1)$ is motivated by Eq. (23), which shows that the normalized Fourier coefficient $f_{m, n} / R(f)_{m}$ has no term linear in $\rho$ for $\rho \ll 1$. This is correct when $e_{f}(m)-\min \left[\bar{m}, e_{f}(m)\right]$ is even, so that 
the expansion Eq. (23) is valid. When this differ-tıce is odd, a discretization based on an expansion of the form $f_{m, n}^{1} \rho+f_{m, n}^{3} \rho^{3}+\cdots$ is requireu. $A$ onesided derivative at $\rho=1$ is used since we assime that values of fields are not. known exterior to the domain $D$.

We have also tried defining $\delta_{\rho}$ to be the operation of differentiating that cubic spline which interpolates the normalized Fourjer coefficient

$$
f_{m, n}(p) / \bar{R}(f)_{m}(p),
$$

using "not-a-knot" boundary conditions ${ }^{27}$ at $\rho=0$ and at $\rho=1$. Here $\bar{R}(f)_{m}$ is a modified radial factor in which one less than the full radial power is used if that power is positive:

$$
\bar{R}(f)_{m}(\rho)= \begin{cases}1 & \text { if } e_{f}(m)=0, \\ \rho^{\min \left(e_{\rho}(m)-1, \bar{m}\right)} & \text { if } e_{f}(m)>0 .\end{cases}
$$

With this definition, Eq. (27) is always bounded and is 0 at $p=0$ for $e_{f}(m)>$ 0 , which facilitates fitting a spline. Differentiating the interpolating spline provides a natural way to obtain derivatives at both integer and half-integer mesh points, and also guarantees that the derivatives are smooth. It has the disadvantage of coupling more points together, and making the derivative more sensitive to small radial fiuctuations in a Fourier coefficient. Empirical numerical studies (see Section $V$ ) show that this alternative definition leads to no significant improvement over finite differences in calculating the metric elements.

\section{Products of Fourier Series}

With the above definitions for derivatives, we can go back to Eqs. (18)(20), calculate the Fourier series of the basis vectors, and combine these basis vectors to obtain the covariant metric elements:

$$
g_{i j}=\mathbf{X}_{, i} \cdot \mathbf{X}_{, j}
$$

This requires determining the Fourier coefficients of products of Fourier series. For example, the metric element

$$
g_{p p}=\left(x_{, p}\right)^{2}+\left(y_{, \rho}\right)^{2}
$$


requires the product of $x_{1 \rho}$ with itself. Products of Fourier series arise in other parts of the PIES code. The Jacobian,

$$
\begin{aligned}
\mathcal{J}^{\rho} & =\frac{\partial(X, Y, Z)}{\partial(\rho, \theta, \phi)}=\mathbf{e}_{\rho} \times \mathbf{e}_{\theta} \cdot \mathbf{e}_{\phi} \\
& = \begin{cases}x\left(x_{, \rho} y_{, \theta}-x_{, \theta} y_{, \rho}\right) & \text { finite aspect ratio } \\
R\left(x_{, \rho} y_{, \theta}-x_{, \theta}, \rho\right. & \text { infinite aspect ratio, }\end{cases}
\end{aligned}
$$

involves products of three Fourier series. The solution of Ampère's law requires the Fourier series of metric elements $\mathcal{J}^{\rho} g^{i j}$; which in turn involves the product of five Fourier series, starting from the coordinates. For example,

$$
\mathcal{J}^{\rho} g^{\rho \rho}=\left(1 / \mathcal{J}^{\rho}\right)\left(g_{\theta \theta} g_{\phi \phi}-g_{\phi \theta} g_{\theta \phi}\right)
$$

with similar formulas for the other terms. From this expression, we see that we also have to work out the Fourier series of the reciprocal of a given Fourier series, e.g., $1 / \mathcal{J}^{p}$.

It was fourd empirically that the effort required in each iteration to transform scalar fields into magnetic coordinates and to solve Ampère's iaw was so large that the computational efficiency of fast Fourier transforms (FFT's) was not needed to calculate products of Fourier series. Instead, we calculated product: of Fourier series by directly convoluting their Fourier coefficients using vertorized subroutines.

To avoid aliasing errors, ${ }^{28}$ multiple products of Fourier series as in Eq. (30) were broken up into repeated pair-wise products. The inverse of a Fourier series was obtained by explicity factoring and inverting convolution equations. For each radjal surface, we solved for the $(M+1)(2 \mathcal{N}+1) \times(\mathcal{M}+1)(2 N+1)$ matrix $M_{m^{\prime}, n^{\prime}}^{m, n}=(1 / f)_{m-m^{\prime}, n-n^{\prime}}^{l}$ that arises from the convolution equations:

$$
\sum_{m^{\prime}, n^{\prime}}(1 / f)_{m-m^{\prime}, n-n^{\prime}}^{l} f_{m^{\prime}, n^{\prime}}^{l}=\delta_{m, 0} \delta_{n, 0} .
$$

This matrix is singular because of the redundancy previously observed for the $m=0$ modes, Eq. (11). A simple preprocessing step eliminates this singular behavior by forcing $f_{0, n}=0$ for $r<0$ before inverting the matrix, then restoring the symmetry afterwards. Although more expensive than using FFT's, solving Eq. (31) gives an exact inverse $(1 / f)_{\mathrm{m}, \mathrm{n}}^{l}$ for given angular resolutions $m$ and $n$. Again, the extra expense is acceptable since other parts of the code dominate the CPU time. 


\section{The Nonvariational Method}

In this section, we explain the details of the our algorithm: how equilibrium solutions to the MHD equations can be chtained by iterating these equations in carefully chosen coordinate systems. We first discuss how the force balance equation, Eq. (1), can be reduced to a magnetic differential equation for $\nu$, the stream function for the current density. ${ }^{6,29}$ Then we discuss how Ampère's law can be solved to obtain a field h whose curl gives this current. ${ }^{5}$ This field is generally not solenoidal but can be made so by adding the gradient of a scalar field $u$. We next discuss how to obtain $u$, which requires the solution of a Poisson equation with Neumann boundary conditions in a nonseparable coordinate system. Finally, we discuss blending and filtering of Fourier coefficients, which are sometimes needed to improve convergence. The reader may wish to follow the analytic example in Appendix $A$ to get a clearer understanding of the algorithm, and to verify the different steps as they are discussed.

\section{A. Boundary Conditions and Surface Functions}

We want to solve the MHD equations, Eqs. (1)-(3), in the dornain defined by Eq. (5). The MHD equations have to be supplemented with boundary conditions and by two surface functions (or profiles). ${ }^{30}$ As discussed earlier, we assume a fixed perfectly conducting boundary so that the outermost magnetic surface is known:

$$
\text { B } \cdot \nabla \rho=B^{\rho}=0, \quad \text { for } \rho=1 .
$$

This boundary condition enters the Poisson equation related to Ampère's law, and implies a Neumann boundary condition (see Section D).

A second boundary condition is also required in sotving Ampère's law t. pin down the multivaluedness of the scalar solution to the Poisson equation (this is needed since the domain $D$ is generally not simply connected and a Neumann boundary condition does not specify a urique solution). There are two natural boundary conditions that can be imposed: conservation of net toroidal flux

$$
\int \mathbf{B} \cdot d \mathbf{S}_{\phi}=\int_{\phi=\phi_{0}} \mathbf{B} \cdot\left(\mathcal{J}^{\rho} \nabla \phi\right) d \rho d \theta=\text { constant }
$$


where the integration goes over a surface of constant toroidal angle $\phi=\phi_{0}$ that intersects the domain; or conservation of net external poloidal current (i.e., current through the external coils that thread the torus):

$$
\int \mathbf{J} \cdot d \mathbf{S}_{\theta}=\int \mathbf{B} \cdot d \mathbf{d}_{\phi}=\int_{\rho=1, \theta=\theta_{0}} \mathbf{B} \cdot\left(\mathcal{J}^{\circ} \nabla \rho \times \nabla \theta\right) d \phi=\text { constant, }
$$

where the integration now goes over the boundary of the interior hole of the domain. Equation (33) involves an integration over $\rho$, which couples all the radial surfaces together. Equation (34) couples only the outermost three radial surfaces and leads to a nearly block tridiagonal matrix for the Poisson equation. This is more convenient numerically. It is also physically more similar to the conditions of many experiments.

Besides boundary conditions, two surface functions must be specified in order to obtain a unique solution to the MHD equations. ${ }^{30}$ It is convenient to specify the pressure profile $p(\rho)$ and a current profile, either the net toroidal current $I(\rho)$ or the net poloidal current $g(\rho)$.

The PIES code therefore begins with an initial magnetic field $\mathbf{B}$, a pressure profile $p(\rho)$, and a current profile $[I(\rho)$ or $g(\rho)]$. The contravariant components of the magnetic field are first given in the usual pseudo-torojdal coordinate system. The fields $x, y$ and $B^{\phi}$ are evaluated along $L+1$ field lines as functions of $\phi$ by integrating the equations:

$$
\frac{d \rho}{d \phi}=\frac{B^{\rho}(\rho, \theta, \phi)}{B^{\phi}(\rho, \theta, \phi)} ; \quad \frac{d \theta}{d \phi}=\frac{B^{\theta}(\rho, \theta, \phi)}{B^{\phi}(\rho, \theta, \phi)},
$$

where we make the important assumption that $B^{\phi}$ is nonzero in the toroidal domain. The $L+1$ starting points for the field lines define a uniformly spaced radial mesh along a symmetry axis connecting the magnetic axis $\rho=0$ and the boundary $\rho=1$. This symmetry axis exists by our assumption of the stellarator symmetry, Eq. (6).

Applying the algorithm of Ref. 6, we deduce the Fourier expansions in magnetic coordinates $(\rho, \theta, \phi)$ of the fields $x, y$, and $B^{\phi}$. The algorithm also yields the rotational transform $t(\rho)$, which is the winding number of the field line in the magnetic surface defined by that field line. The Fourier coeff. cients of all these quantities are determined by the line-following algorithm to an accuracy $f$ tprec which is an input parameter, typically of order $10^{-6}$. The total external poloidal current, Eq. (34), is held constant during each 
iteration. The physics data needed by the code are summarized in Table 11, while the numerical data are summarized in Table 111.

Starting with these Fourier expansions in magnetic coordinates, we can then proceed to the iteration of the MHD equations, Eqs. (1)-(3). It is usefu' in what follows to use a different radial variable than $\rho$. The new radial variable $\psi$ arises naturally from the canonical form of a magnetic field that has magnetic surfaces ${ }^{30}$ :

$$
\mathbf{B}=\nabla \psi \times \nabla \theta+\imath \nabla \phi \times \nabla \psi
$$

Here $\psi(\rho)$ is a nonnegatjve monotonic function of the radial variable $\rho$; it is easily seen that $2 \pi \psi$ is the toroidal flux. The rotational transform $t(p)$ is the surface quantity mentioned previously, giving the average rate of poloidal rotation of the field line.

We can use $\psi$ as a radial variabje provided we know the relation $\psi(\rho)$. This function can be determined on existing magnetic surfaces by averaging the ratio of the Jacobians between Cartesian and magnetic coordinates, with $\rho$ and $\psi$ as independent radjal variables respectively. As discussed in Section III, the $\rho$-Jacobian, Eq. (29), is known in terms of derivatives and convolutions of the cylindrical coordinates $x$ and $y$. From Eq. (35) we see that the Jacobian between magnetic coordinates with $\psi$ as the independent variable is determined by the $B^{\phi}$ component of the magnetic field:

$$
\begin{aligned}
\mathcal{J}^{\psi} & =\frac{\partial(X, Y, Z)}{\partial(\psi, \theta, \phi)}=\frac{1}{\nabla \psi \times \nabla \sigma \cdot \nabla \phi}=\frac{1}{\mathbf{B} \cdot \nabla \phi}=\frac{1}{B^{\phi}} \\
& =\sum_{m, n} \mathcal{J}_{m, n}^{\psi}(\psi) \cos (n \phi-m \theta) .
\end{aligned}
$$

The Fourier coefficients of $1 / B^{\phi}(\psi, \theta, \phi)$ are known by having evaluated $B^{\phi}$ along field lines of $\mathbf{B}$, using the transformation to magnetic coordinates of Ref, 6, and inverting the Fourier matrix, Eq. (31). The averaged ratio of the two Jacobians then gives:

$$
\frac{d \psi}{d \rho}=\left\langle\mathcal{J}^{\rho}\left(1 / \mathcal{J}^{\psi}\right)\right\rangle=\left\langle\mathcal{J}^{\rho} B^{\phi}\right\rangle
$$

where the brackets $(\ldots)$ denote an average over poloidal and toroidal angles. We see that $\dot{\psi}^{\prime}(p)$ is the $m=n=0$ mode of $\mathcal{J}^{\circ} B^{\phi}$. 


\section{B. Calculation of the Current Density $\mathbf{J}$}

From $\nabla \times \mathbf{B}=\mathbf{J}$, we deduce that the current density $\mathbf{J}$ must be solenoidal, ard, from $\mathbf{J} \times \mathbf{B}=\nabla p$ we see that $\mathbf{J}$ must lie on magnetic surfaces. ${ }^{30}$ The current density can therefore also be represented in a canonical form similar to Eq. (35):

$$
\mathbf{J}=\left(I^{\prime}(\psi)-\frac{\partial \nu}{\partial \theta}\right) \nabla \psi \times \nabla \theta+\left(\frac{\partial \nu}{\partial \phi}-g^{\prime}(\psi)\right) \nabla \phi \times \nabla \psi
$$

where we are now using $\psi$ as the radial variable. The single-valued scalar $\nu(\psi, \theta, \phi)$ is a degree of freedom that is allowed under the canstraints $\nabla \cdot \mathbf{J}=\mathbf{0}$ and $\mathbf{J} \cdot \nabla \psi=0$. By iniegrating $\mathbf{J} \cdot \nabla \phi$ over the volume enclosed by a magnetic surface labeled by $\psi$, we see that $2 \pi I(\psi)$ is the net toroidal current profile. Similarly, integrating the quantity $\mathrm{J} \cdot \nabla \theta$ over a surface labeled by $\theta$ shows that $2 \pi g(\psi)$ is the net poloidal current profile external to the magnetic surface.

We can derive an equation for $\nu(\psi, \theta, \phi)$ by substituting Eqs. (35) and (38) into the force balance equation, $\mathrm{Eq}$. (1). This yields the following magnetic differential equation ${ }^{29}$ :

$$
(\mathbf{B} \cdot \nabla) \nu=\frac{g^{\prime}+t I^{\prime}}{J^{b}}+\frac{d p}{d \psi}
$$

or

$$
\left(\frac{\partial}{\partial \phi}+t \frac{\partial}{\partial \theta}\right) \nu=g^{\prime}+t I^{\prime}+\frac{d p}{d \psi} J^{\psi}
$$

where the ' denotes differentiation with respect to $\psi$. If we integrate both sides of this equation over $\theta$ and $\phi$ and use the fact that $\nu$ is single-valued and hence periodic, we see that Eq. (39) hes a solution only if

$$
g^{\prime}+t I^{\prime}+\frac{d p}{d \psi} \mathcal{J}_{0,0}=0
$$

where $\mathcal{J}^{\psi}{ }_{0,0}$ is the $m=n=0$ mode of the Fourier representation of $\mathcal{J}^{*}$ [see Eg. (36)|. This relation allows us to specify either $I^{\prime}$ or $g^{\prime}$ and to solve for the other current profile. This relation between the two profiles was originally given by Kruskal and Kulsrud ${ }^{30}$ in the form $d p d V=d \chi d I-d \psi d g$, where $V$ is the volume enclosed by a magnetic surface labeled by $\psi$. 
We can write down the solution to the equation for $\nu$, Eq. (39), in terms of the Fourier coefficients of $\mathcal{J}^{\psi}[\mathrm{Eq} .(36)]$ :

$$
\nu(\psi, \theta, \phi)=\frac{d p}{d \psi} \sum_{m, r^{n}}^{\prime} \frac{\mathcal{J}^{\psi} m_{m, n}}{(n-t m)} \sin (n \phi-m \theta)
$$

where the ' indicates that the $m=n=0$ term is omitted. Substituting this into the expression for $\mathbf{J}$, Eq. (38), we get the following canonical expression for the current density:

$$
\begin{aligned}
\mathbf{J} & =\nabla \psi \times \nabla \theta\left(I^{\prime}(\psi)+\frac{d p}{d \psi} \sum_{m, n}^{\prime} \frac{m J^{\psi}{ }_{m, n}}{(n-t m)} \cos (\pi \phi-m \theta)\right) \\
& +\nabla \phi \times \nabla \psi\left(-g^{\prime}(\psi)+\frac{d p}{d \psi} \sum_{m, n}^{\prime} \frac{n J^{\psi}{ }_{m, n}}{(n-t m)} \cos (n \phi-m \theta)\right)
\end{aligned}
$$

Together with Eq. (40), this finishes the first step of the PIES algor thm, calculating the total current density $\mathbf{J}$ in terms of $\mathbf{B}$, the pressure profile $p^{\prime}$, a current profile, and a known outermost flux surface.

\section{Solution of Ampère's Law}

We next discuss how to solve Ampère's law, Eq. (2), for a new solenoidal magnetic field $b$. (We use lower case symbols to represent new fields at the end of one iteration.) As Boozer observed, ${ }^{5}$ it is simple to find a field $h$ such that $\nabla \times h=J$. In fact, if $h$ is not solenoidal, there is an infinity of such

fields that all differ by the gradient of some scalar. Ampère's law in covariant. form leads to the following three equations on each radial surface:

$$
\begin{aligned}
& n\left(h_{\theta}\right)_{m, n}+m\left(h_{\phi}\right)_{m, n}=\left(\mathcal{J}^{\rho} J^{\rho}\right)_{m, n}, \\
& n\left(h_{\rho}\right)_{m, n}-\partial_{\rho}\left(h_{\phi}\right)_{m, n}=\left(\mathcal{J}^{\rho} J^{\theta}\right)_{m, n}, \\
& m\left(h_{\rho}\right)_{m, n}+\partial_{\rho}\left(h_{\theta}\right)_{m, n}=\left(\mathcal{J}^{\rho} J^{\phi}\right)_{m, n}
\end{aligned}
$$

This suggest two natural gauges. First, we can set one of the angular comporents, $h_{\theta}$ or $h_{\phi}$, to zero for $m>0$. This gives the following solution (which 
generalizes Boover's gauge ${ }^{5}$ to the case of finite $J^{p}$ ):

$$
\begin{aligned}
& \left\langle\left(h_{p}\right)_{m, n},\left(h_{\theta}\right)_{m, n},\left(h_{\phi}\right)_{m, n}\right\rangle= \\
& \quad \begin{cases}\frac{2}{m}\left\langle\mathcal{J}^{\rho} J_{m, n}^{\phi}, 0, \mathcal{J}^{\rho} J_{m, n}^{\rho}\right\rangle & \text { for } m>0 ; \\
\frac{1}{n}\left\langle\mathcal{J}^{\rho} J_{m, n}^{\theta}, \mathcal{J}^{\rho} J_{m, n}^{\rho}, 0\right\rangle & \text { for } m=0 \text { and } n \neq 0 ; \\
\left\langle 0, \int_{0}^{\rho} \mathcal{J}^{\rho} J_{0,0}^{\phi} d \rho,-\int_{0}^{\rho} \mathcal{J}^{\rho} J_{0,0}^{\theta} d \rho\right\rangle & \text { for } m=n=0 .\end{cases}
\end{aligned}
$$

For fields with magnetic surfaces [Eq. (42)], wr: can write this out explicitly:

$$
\begin{aligned}
\mathbf{h}= & \left(\frac{d p}{d \rho} \sum_{m, n}^{\prime} \frac{J_{m, n}^{\psi}}{(n-t m)} \sin (n \phi-m \theta)\right) \nabla \rho \\
& +[I(\rho)-I(0)] \nabla \theta+[g(\rho)-g(0)] \nabla \phi .
\end{aligned}
$$

Another choice of gauge is $\left(h_{p}\right)_{m, n}=0$ for all $m$ and $n$, which gives the following solution to Ampère's law:

$$
\begin{aligned}
& \left\langle\left(h_{\rho}\right)_{m, n},\left(h_{\theta}\right)_{m, n},\left(h_{\phi}\right)_{m, n}\right\rangle= \\
& \quad\left\langle 0, \int_{0}^{\rho} \mathcal{J}^{\rho} J_{m, n}^{\phi} d \rho,-\int_{0}^{\rho} \mathcal{J}^{\rho} J_{m, n}^{\theta} \dot{\alpha \rho}\right\rangle .
\end{aligned}
$$

For fields with magnetic surfaces, we can again write this out explicitly:

$$
\begin{aligned}
\mathbf{h} & =\left[I(\rho)-I(0)+\sum_{m, n}^{\prime} m \int_{0}^{\rho}\left(\frac{d p}{d \rho} \frac{\mathcal{J}_{m, n}^{\psi}}{n-t m}\right) d \rho \cos (n \phi-m \theta)\right] \nabla \theta \\
& +\left[g(\rho)-g(0)-\sum_{m, n}^{\prime} n \int_{0}^{\rho}\left(\frac{d p}{d \rho} \frac{J^{\psi} m, n}{n-t m}\right) d \rho \cos (n \phi-m \theta)\right] \nabla \phi .
\end{aligned}
$$

For circular magnetic surfaces, this gives $h^{\rho}=0$, so that this gauge is appropriate for equilibria with nearly circular magnetic surfaces. Although both gauges are analytically equivalent in that both satisfy $\nabla \times \mathbf{h}=\mathrm{J}$, they are different numerically. In particular, Eq. (47) involves only the radial integrals of $m=n=0$ modes, while Eq. (49) involves radial integrals of higher mode numbers.

For values of $\rho$ corresponding to magnetic islands or stochastic regions, it is a reasonable first approximation to assusic force-free equilibria, $\mathbf{J}=\mu \mathbf{B}$, where $\mu(\psi)$, the force-free current profile, is constant along a field line in 
the jsland. The Fourier coefficients for this new expression for $\mathbf{J}$ can be substituted directly into Eqs. (46) or (48), which gives $h$ in the island. There are further subtleties involving the derivation of a criterion for identifying field lines that lie in islands or stochastic regions and interpolation of the coordinate system through the magnetic islands. These are discussed in Ref. 6.

Both choices of gauge for h require numerical approximations to integrals of Fourier coefficients. We tried two different numerical approximations in order to test the sensitivity of the code to the accuracy of $\mathbf{h}$. The first method was the usual trapezoidal rule. The second method evaluated integrals by analytically integrating the interpolating splines of the integrands, using "not-a-knot" boundary conditions" at $\rho=0$ and at $\rho=1$. Numerical experiments showed that the convergence of the PIES code was only weakly dependent on the choice of integration algorithm. In particular, the trapezoidal rule gave satisfactory results. This is an important observation when the code is used for equilibria with islands, since local radial information of the Fourier coefficients suffices to estimate the integral.

A potential difficulty with solving directly for $h$ in Ampère's law is that this field remains of $O(1)$ as the iterations proceed. This could lead to numerical convergence problems since, as we will see in the next section, the $\rho$ component of the new magnetic field has the form:

$$
\mathcal{s}^{\boldsymbol{O}}=\nabla_{\boldsymbol{\rho}} \cdot(\mathbf{h}+\nabla u+\lambda \nabla \phi)
$$

which must vanish on the outermost magnetic surface, $p=1$. Here $\nabla u+\lambda \nabla \phi$ is needed to make $h$ divergence free and to satisfy the boundary condition Eq. (34).

If $\mathbf{h}$ remains of $O(1)$, then the scalar $u$ also remains of $O(1)$, and a large cancellation must take place to allow $b^{\circ}$ to ranish. This cancellation could magnify numerical noise and slow convergence. We therefore explored a modification of the PIES algorithm in which the $\mathbf{B}$ field at the beginning of the iteration was subtracted from the right side of Ampere's law:

$$
\nabla \times \mathbf{h}=\mathbf{J}-\nabla \times \mathbf{B},
$$

so that $\mathbf{h}$ becomes the correction to the magnetic field from the previous iteration. In particular, $h$ and $u$ now go to zero as the code converges. 
Appendix $B$ discusses how the magnetic field $B$ can be expressed in terms of the its own magnetic coordinates, so that $\nabla \times \mathbf{B}$ in Eq. (50) is a meaningful expression. The above expressions for the components of $\mathbf{h}$ can then be modified to subtract off this new field. For example, for the generalized Boozer gauge, Eq. (46), the $m>0$ terms become:

$$
\begin{aligned}
& \left(h_{p}\right)_{m, n}=\frac{1}{m}\left(\mathcal{J}^{\rho} J_{m, n}^{\phi}-m\left(b_{\rho}\right)_{m, n}-\partial_{\rho}\left(b_{\theta}\right)_{m, n}\right), \\
& \left(h_{\theta}\right)_{m, n}=0, \\
& \left(h_{\phi}\right)_{m, n}=\frac{1}{m}\left(\mathcal{J}^{\rho} J_{m, n}^{p}-m\left(b_{\phi}\right)_{m, n}-n\left(b_{\theta}\right)_{m, n}\right) .
\end{aligned}
$$

The radial derivative $\partial_{\mathcal{\rho}}\left(b_{\theta}\right)$ is calculated using leading radial factors as described in Eq. (25). The other components are treated in a similar way. The consequence of subtracting $\nabla \times B$ is discussed in Section V.

\section{Solving a Poisson Equation for $u$}

The field $h$ of the previous section is typically not divergence-free and so does not satisfy the last of the MHD equations, Eq. (3). It can be made solenojdal-without changing its curl-by adding the gradient of some scalar. A magnetic field $b$ whose curl is $\mathbf{J}$ is then given by:

$$
\mathbf{b}=\mathbf{h}+\nabla \boldsymbol{u}+\lambda \nabla \boldsymbol{\phi},
$$

where $u$ is a single-valued scalar field to be determined, and where the constant $\lambda$ fixes the possible multivalued correction to $u$ and is determined by the conservation of the total external poloidal current, Eq. (34):

$$
\int_{\rho=1, \theta=\theta_{0}} B^{0} \cdot d l=\int_{p=1, \theta=\theta_{0}}(h+\nabla u+\lambda \nabla \phi) \cdot d \boldsymbol{l} .
$$

The integrais go over a closed loop on the outermost magnetic surface and are independent of $\theta_{0}$ provided there is no net poloidal current on the outermost magnetic surface. Since $u$ is single-valued, the line integral over a closed loop of its gradient is zero. The left side is known from the injtial field $\mathbf{B}^{\circ}$ and $\mathbf{h}$ is known from Eq. (47) or from Eq. (49). We then find

$$
\lambda=\left(B_{\phi}^{0}\right)_{0,0}^{l}-\left(h_{\phi}\right)_{0,0}^{l}
$$


which involves the $m=n=0$ Fourier components of $B_{\phi}^{0}$ and $h_{\phi}$ at $\rho=1$ $\{l=L)$.

The requirement that Eq. (51) satisfies Eq. (3) leads to a Poisson equation for $u$ :

$$
\nabla^{2} u=-\nabla \cdot(\mathbf{h}+\lambda \nabla \phi) .
$$

The boundary condition is determined by our assumption of a fixed perfectly conducting boundary, $b^{p}=0$ for $\rho=1$, Eq. (32). This leads to a Neumam condition on $u$ :

$$
\nabla \rho \cdot \nabla u=-(\mathbf{h}+\lambda \nabla \phi) \cdot \nabla \rho \quad \text { for } \hat{\rho}=1 .
$$

Equations (53) and (54) have to be solved numerically ior the Fourier coefficjents of $u$ on each magnetic surface. A solution always exists since the usual integral condition for the existence of a solution $u$ of a Poisson equation with a Neumann boundary condition ${ }^{31}$ is trivially satisfied: the volume integral of the right side of Eq. (53) is equal to the surface integral of the right side of Eq. (54).

The retention of the $\nabla \phi$ term on the right side of Eq. (53) requires some explanation. Although $\phi$ is harmonic, so that $\nabla \cdot \nabla \phi=\nabla^{2} \phi=0$, the discrete operator approximating the Laplacian does not gener $5 y$ kjll $\phi$. We found that retaining the $\nabla \phi$ term on the right side increased the numerical stability of the PIES code. The reason is probably that a similar gradient term appears in Eq. (54), so that the right side of the discrete equations in the interior of the domain is more consistent with the right side of the boundary condition.

The details of how Eqs. (53) and (54) are discretized and solved are given in a forthcoming publication. As is pointed out in that paper, the main subtlety is choosing a discretization that is numerically stable when used in the larger context of the PIES algorithm. By using a conservative discretization of the Laplacian, rapidly growing instabilities near the outermost magnetic surface can be eliminated. The traditionally difficult region near the magnetic axis can be controlled by using leading radial factors when performing derivatives or averages. A weak slowly growing radial instability sometimes remains, but can be eliminated by blending or filtering of Fourier coefficients, as discussed in the next section. 


\section{E. Blending and Filtering of Fields}

This completes one cycle of iterating through the $\mathrm{NH}$ HD equations. In general, many cycles are needed, the exact number depending on the choice of initial conditions, the numerical resolution, the desired accuracy, and the values of physical parameters. Since the PIES algorithm is a Picard iteration scheme, in which the new fields are directiy fed back 27 input to the next cycle of iteration, convergence can be slow or even unstable without some massaging of the fields before entering the next iteration. We have found it sometimes useful to blend the Fourier coefficients at the end of one iteration with those of the previous iteration (which aids convergence by preventing ascillation or overshooting). It is also sometimes useful to filter certain Fourier coefficients radially. This eliminates a weak instability of high-frequency noise that can appear after many jterations.

Since the coordinates and the representations of fields ate changing at each iteration with $\mathbf{B}$, it is important to choose fields for blending that are invariant under coordinate transformations. This results in smaller changes of the radial dependence of Fourier coefficients with succeeding iterations. A careful examination of the algorithm presented in Section IV suggests that the most useful fields for blending are:

$$
B^{\phi}(\rho, \theta, \phi), \quad(\rho, \theta, \phi), \quad y(\rho, \theta, \phi), \quad \text { and } t(\rho) .
$$

This is roughly equivalent to blending the magnetic field since $\mathbf{B}$ is known in terms of these quantities:

$$
\mathbf{B}=B^{\phi}\left(\frac{\partial \mathbf{X}}{\partial \phi}+t \frac{\partial \mathbf{X}}{\partial \theta}\right),
$$

which is the expression for $B$ in covariant basis vectors [Eq. (35)!, using the fact that $1 / \mathcal{J}^{\psi}=B^{\phi}$ by Eq. (36), and using Eqs. (12)-(17) to express $\mathrm{X}$ in terms of $x$ and $y$.

We introduce three different blending coefficients for these fields: bblnd, ryblnd, and iblad, and blend the Fourier coefficients at the latest iteration $(K+1)$ with those of the previous one $(K)$, using a simple reiaxation scheme:

$$
\begin{aligned}
B^{\phi}(K+1) & =B^{\phi}(K \perp 1)+\operatorname{bblnd}\left[B^{\phi}(K)-B^{\phi}(K+1)\right], \\
\mathbf{x}(K+1) & =\mathbf{x}(K+1)+\operatorname{xyblnd}[\mathbf{x}(K)-\mathbf{x}(K+1)], \\
t(K+1) & =t(K+1)+\operatorname{ib} \operatorname{lnd}\{t(K)-t(K+1)] .
\end{aligned}
$$


It is understood that the blending is applied to each separate Fourier coefficient. More sophisticated blending algorithms could also be used that give faster convergence by using information from earlier iterations. ${ }^{32}$

In addition to blending, we also implemented a subroutine for radial filtering of the Fourier coefficients of certain fields such as $\mathbf{X}$ and $B$. As described in Section V, this is sometimes required since the PIES algorithm can become unstable to high-frequency radial modes after many iterations. One simple method for removing this high-frequency noise has been described by Shapiro. ${ }^{33}$ The idea is to use a radial diffusion operator to eliminate the bighest frequency noise, and then apply an "antidiffusion" operator to restore the amplitudes of the lower frequency radial modes. If we define the operator

$$
F_{s}\left[f_{m, n}^{l}\right]=(1-s) f_{m, n}^{l}+\frac{s}{2}\left(f_{m, n}^{l+1}+f_{m, n}^{l-1}\right),
$$

$t^{2}$.en $F$, is diffusive for $s>0$ and antidiffusive for $s<0$. Following Shapiro, we use a two-piece filter on each Fourier coefficient:

$$
\bar{f}_{m, n}^{l}=F_{s 2}\left[F_{s 1}\left[f_{m, n}^{l}\right]\right],
$$

It was shown by Shapiro that the values $s 1=1 / 2$ and $s 2=-1 / 2$ are optimal choices for these parameters in that the highest frequency mode is removed with minimal amplitude or phase change of smaller frequency radial modes, and that no other linear filter acting on five neighboring points can do as well. ${ }^{33}$ Our experience is that weak damping usually suffices, with the antidiffusing slightly improving convergence.

Equation (60) has to be modified near $\rho=0$ and $\rho=1$ so that the filtering is consistent with known boundary conditions on the Fourier coeffcients. These boundary conditions are that the correct analytic behavior be recovered by the filtered coefficients, and that information on the outermost magnetic surface is assumed to be more accurate than interior information. Two modifications are necessary. First, instead of filtering the Fourier coefficjents directly, we pull out a leading radjal power, with one less that the full power for $m>0$ so that the "normalized" coefficient vanishes at the magnetic axis. We then filter the "normalized" Fourier coefficient, and then restore the radial factor. This helps to preserve the correct leading radial behavior near $\rho=0$, which would be otherwise lost if the filtering were directly applied. Second, we modify the weighting of neighboring terms in the filter 
Eq. (60) so that the highest frequency mode at $\rho=d \rho$ and at $\rho=1-d \rho$ is killed in one application of the filter. It should be further noted that care must be used when filtering divergence-free fields such as $\mathbf{B}$, since the filtered fields are not necessarily divergence-free.

The filter requires as input to the PIES code three parameters: fltrent, which determines how many times the filter Eq. (60) is applied in succession to each Fourier coefficient during a given iteration; 81, which determines the amount of radial diffusion; and $\mathbf{s 2}$ which determines the amount of radial antidiffusion.

\section{Results}

In this section we discuss the results of numerical experiments in which we apply the PIES code to two problems: an axisymmetric tokamak equilibrium (the analytic Solov'ev solution ${ }^{34}$ ), and a nonaxisymmetric finite $\beta$ stellarator equilibrium. The latter is chosen to have a small rotational transform to avoid complications arising from resonances. In each case, we present plots of various Fourier coefficients as functions of the radius and study how the convergence of the code depends on the physical parameters of Table II, and on the numerical parameters of Table III.

The convergence rate is measured by calculating at the end of each iteration the magnitude of the residual of the MHD equilibriurn equations, Eqs. (1)-(3). The residual is defined to be a maximum norm of the force balance expression, $\mathbf{J} \times \mathbf{B}-\nabla p$, normalized to the maximum pressure gradient (the latter is set to one for force-free equilibria):

$$
\begin{aligned}
\text { residual }= & \frac{1}{\left\|p^{\prime}(\rho)\right\|} \max \left\{\left\|\mathcal{J}^{\rho}\left(J^{\theta} B^{\phi}-J^{\phi} B^{\theta}\right)-p^{\prime}(\rho)\right\|,\right. \\
& \left.\left\|\mathcal{J}^{\rho}\left(J^{\phi} B^{\rho}-J^{\rho} B^{\phi}\right)\right\|,\left\|\mathcal{J}^{\rho}\left(J^{\rho} B^{\theta}-J^{\theta} B^{\rho}\right)\right\|\right\} .
\end{aligned}
$$

This expression tends to zero as convergence is attained. The maximum norm of a Fourier series is defined to be:

$$
\left\|\sum_{m, n} f_{m, n}(\rho) \sin (n \phi-m \theta)\right\|=\frac{1}{(m+1)(2 n+1)} \max _{m, n, l}\left|f_{m, n}^{l}\right| .
$$

Since the current density $J(K)$ at the $K$ th iteration is determined from $B(K)$ by force balance, the residual is only a useful criterion for convergence when 
the new magnetic field $\mathbf{B}(K+1)$ from Eq. (51) is used in evaluating the residual.

We emphasize that the following results are numerical experiments and that the PIES code has a high-dimensional numerical parameter space that is difficulh to characterize with a moderate number of runs. Our results illustrate how the code behaves for a tokamak and a stellarator equilibrium, which we hope (but can not demonstrate) to be representative.

\section{A. Axisymmetric Tokamak Equilibrium}

A test that is commonly used to verify equilibrium codes is the Solov'ev analytic tokamak equilibrium. ${ }^{34}$ An axisymmetric field can be written in the form

$$
\mathbf{B}=\nabla \psi_{\mathrm{p}} \times \nabla \phi+g \nabla \phi,
$$

where $g\left(\psi_{p}\right)$ is the polojdal current and $\psi_{p}$ is the poloidal flux enclosed by a given magnetic surface. This satisfies the MHD equations, Eqs. (1)-(3), provided $\psi_{p}$ satisfies the Grad-Shafranov equation. ${ }^{35}$ In cylindrical coordinates $(\tau, z, \phi)$, an analytic solution is given by

$$
\psi_{p}(r, z)=\psi_{0}\left(\frac{r}{R}\right)^{2}\left[2-\left(\frac{r}{R}\right)^{2}-4 \alpha^{2}\left(\frac{z}{R}\right)^{2}\right],
$$

with

$$
\begin{aligned}
p^{\prime}(\psi) & =\frac{8\left(1+\alpha^{2}\right) \psi_{0}}{R^{4}}=\text { constant } \\
g & =g_{0}=\text { constant }
\end{aligned}
$$

where $R$ is the major radius of the torus and $g_{0}, \psi_{0}$, and $\alpha$ are arbitrary parameters.

These parameters can be chosen as follows. If we normalize $\mathbf{B} \cdot \nabla \phi=1$, the parameter $g_{0}$ should be of order $R^{2}$ so we set $g_{0}=R^{2}$. (The major radius $R$ is set to 10.) The parameter $\alpha$ determines the shape of the magnetic surfaces and we choose a value $\alpha=1$ that gives nearly circular surfaces. The parameter $\psi_{0}$ determines the rotational transform near the magnetic axis. We have set $\psi_{0}=0.14$ to obtain an axis transform of about 0.14 . The poloidal resolution is fixed at $M=10$. This is effectively infinite poloidal 
resolution since increasing $M$ does not change the results. The convergence properties of the code are then studied as functions of the radial resolution $L$ and of the Fourier coefficient precision ftproc determined by the numerical transformation to magnetic coordinates.

We first test the 'IES code by starting the code with the exact analytic solution, Eqs. (63) and (64), and with the profiles in Eqs. (65) and (66). The residual after one iteration then measures how accurately the code can approximate the analytic answer for given radial and angular resolution, and also determines the overall convergence rate of the code (i.e., the constant coefficient of the error term arising from the finite radial discretization). We see from Fig. 2 that the code achieves quadratic convergence, i.e., that residual $\propto 1 / L^{2}$ for sufficiently large $L$, with a constant of proportionality of about 0.1 . This is a strong check that various finite difference approximations were consistently implemented. The figure also demonstrates that the code is indeed solving the MHD equilibrium equations, i.e., as the resolution increases, the initial exact solution is a fixed point of the discrete equations to better and better accuracy.

We next test the PIES code by perturbing the Solov'ev equilibrium and studying the convergence back towards the equilibrium solution. We add a perturbation of the form

$$
\psi_{1}=\left\{\begin{array}{ll}
\epsilon \rho \cos (\theta)\left(\rho_{0}^{2}-\rho^{2}\right) & \text { for } \rho<\rho_{0}, \\
0 & \text { for } \rho \geq \rho_{0}
\end{array},\right.
$$

to Eq. (64) where the parameters $\epsilon$ and $\rho_{0}$ determine the magnitude of the outward shift of the magnetic axis and the radial limit of the perturbation respectively.

We have chosen values $\epsilon=0.3$ and $\rho_{0}=0.75$. We start the code with the perturbed $B$ field and with the same profiles as before, and iterate until no further changes occur or until the code diverges (the residual starts increasing). Figure 3 shows the $(\rho, \theta)$ grid for the perturbed initial state (a) and the final equilibrium state (b).

A more quantitative understanding of the convergence properties of the code is obtained by systematically varying numerical parameters that affect convergence, the most important of which relate to blending and filtering (see Section E). In Fig. 4, we show how the residual varies with iteration number $K$ for fixed angular and radia! resolutions, and with different com- 
binations of fields being blended [see Eq. (55)]. Figure 4 a has no filtering of Fourier coefficients $(s 1=s 2=0$ ), while for Fig. $4 \mathrm{~b}, \boldsymbol{s 1}=1 / 2$ and $s 1=-1 / 2$.

We see from Fig. $4 \mathrm{a}$ and Fig. $4 \mathrm{~b}$ that convergence is slow or not attainable unless $t(\rho)$ is blended. In the absence of filtering (Fig. 4a), blending $t$ alone is not sufficient for convergence. The code stops after two iterations because of numerical noise near the magnetic axis. We also learn from this figure that filtering has only a moderate affect on convergence, stabilizing the case where only $t$ is blended (-i-) and destabilizing the case where no fields are blended (--) , and otherwise not affecting the convergence rate. Filtering has affected the atiainable residual, presumably because the strong filtering used here has decreased the effective radial resolution. (The effect of radial resolution on the converged residual will be further discussed below.) Other combinations of blending were also tried (e.g., b--, b-x, bi-, and -ix) and were found to give results similar to the curves in Figure 4, i.e., they confirmed the importance of always blending at least the rotational transform.

The peculiar importance of blending $t(p)$ can be understood by a more careful examination of output from the code. It is found that when $t$ is not blended, the transform is oscillating while the residual remains roughly constant (blending of $t$ would then be expected to damp the oscillations). The origin of the oscillations in $t$ is not hard to determine. The change $\delta t(K)=t(K)-t(K-1)$ in transform from one iteration to the next can be seen to satisfy $\delta_{t}(K+1) \propto C / \delta_{t}(K)$, with the constant of proportionality $C \simeq 1$. Thus increases in $\delta_{t}$ in one iteration lead to decreases in the next, and oscillation tends to result. The derivation holds for equilibria with net toroidal current and so oscillations are predicted not to occur for stellarator equilibria with zero net toroidal current, as is confirmed by our calculations below.

The effects of the radial resolution $L$ and the finite Fourier precision ftprec are more carefully examined in Fig. 5 , where the value of the residual achieved after 20 iterations is plotted as a function of radial resolution $L$ for several different values of ftproc. For large values of ftproc, the residual is independent of $L$; for the range of $L$ studied here) since the discretization error, of order $1 / L^{2}$, is much smaller than ftprec. As ftprec gets significantiy smaller than the radial discetization error, one sees quadratic convergence in the residual, until the latter is again of order Ftprec. The quadratic error term has approximately the same coefficient $(0.1)$ as that observed in Fig. 2. 
The changes in two other important quantities in the Solov'ev equilibrium are given in Figs. 6 and 7. Figure 6 shows how the Fourier coefficients of the $x$ variable change during convergence for the perturbed Solov'ev run. Some Fourjer modes are eliminated, and the radial perturbation is smoothed out. The quantity $B^{\rho} / B^{\phi}$ (Fig. 7) measures the deviation of $\rho$ from the magnetic surfaces. In the 0th iteration, $\rho$ deviates from the self-consistent magnetic surfaces because of the perturbation. Upon convergence, self-consistency has been attained to much better than the parameter ftproc $=10^{-5}$. The random structure of the Fourier modes in Fig. $7 \mathrm{~b}$ indicates that convergence has been attained to within the available number of significant digits (ftprec), so that only numerical noise remains.

We conclude that, for tokamak equilibria, some blending (but not filtering) of fields is needed to obtain convergence, which is quadratic for suffciently small ftprec. The code is unstable without blending.

\section{B. Nonaxisymmetric Stellarator Equilibrium}

The second example that we systematically studied was a more challenging nonaxisymmetric stellarator equilibrium. In the absence of axisymmetry, one expects resonances and island formation on rational magnetic surfaces. In practice, one can choose physical parameters (corresponding to a small value of the rotational transform) so that, with low to moderate numerical resolution, resonances do not occur. As the angular resolution increases $(\mathcal{M} \rightarrow \infty, \mathcal{N} \rightarrow \infty)$, resonant denominators will appear and one must treai the magnetic islands and stochastic regions directly [Ref. 3].

We chose a five-period $(N=5) l=2$ stellarator, corresponding to the parameters of Wendelstein VIIA. As is appropriate for stellarators, we imposed zero net toroidal current on each magnetic surface $\left(l^{\prime}=0\right)$. We assumed a pressure profile of the form $p=p_{0}\left(1-p^{2}\right)^{2}$ and a value of $p_{0}=5.4 \times 10^{-3}$, which gives a Shafranov shift of about $20 \%$ of the minor radjus. A Bessel function solution for the vacuum field in a cylinder was used as an initial guess for the field. Thus we set $\mathbf{B}^{0}=\nabla \chi$ where

$$
\chi(\rho, \theta, \phi)=b_{0} \phi+b_{2} I_{2}(N \rho / R) \sin (2 \theta-N \phi) ;
$$

$I_{2}(\rho)$ is a modified Bessel function. Taking $b_{2} / b_{0}=0.667$ gives a relatively low rotationel transform of about $t=0.14$ at the magnetic axis, and also 
yields a relatively low shear. This avoids resonances for moderate angular resolutions.

The convergence properties of the stellarator equilibrium as a function of $L$ and $f$ tprec were found to be similar to those for the Solov'ev equilibrium described in the previous section, and will not be discussed. For this section we will fix $L=20$, fiproc $=10^{-5}$. We also take $M=6$ and $\mathcal{N}=3$. Figure 8 shows the $(\rho, \theta)$ grid for the initial and converged magnetic field. A Shafranov shift of about $20 \%$ is apparent in the converged field.

When the above initial conditions are used without blending of filtering, the residual and corrections are again found to decrease and then increase with successive iterations, indicating some numerical instability of the algorithm. Figure 9 is similar to Fig. 4 in showing how convergenze depends on filtering and blending, with (a) giving the results without filtering and (b) with filtering. The results contrast sharply with the tokamak runs: convergence is not attained for any combination of blending unless filtering is used (the default parameters of $s 1=1 / 2$ and $s 2=-1,2$ were chosen for part (b)]. Even when filtering of Fourier coefficients are turned on, not all combinations of fields being b]ended result in convergence. The runs that do converge seem stable out to the largest iteration numbers studied (about 30). The smallest residual attajned for convergent runs again depends on the parameter ftprec, approaching but never becoming smaller than this value.

Because of the importance of filtering in stellarator runs, we studied more systematically the sensitivity to convergence of the code on the filtering parameters $s 1$ and $s 2$ in Fig. 10. The main conclusion of this figure is the need for some minimal radial smoothing. It is not necessary to entirely remove the highest frequency component $(s 1=1 / 2)$. When complete filtering of the highest frequency componeri $(s 1=1 / 2)$ is employed, a subsequent antidiffucion improves the accuracy of the solution somewhat by restoring the amplitudes of the lower frequency modes.

For insufficient filtering, the code stops because of numerical noise, arising frc:n a numerical instability, appearing near the magnetic axis. This prevents the algorithm used for transforming to magnetic coordinates from finding a magnetic axis. ${ }^{6}$ Some insight into this instability is given by Fig. 11, which shows the radial dependence of the Fourier coefficients of $B^{\rho} / B^{\phi}$ for several iterations. The Fourier coefficients are smooth and well behaved until about the ffth iteration, at which point kinks with magnitude above ftproc appear 
near the magnetic axis $(\rho \leq 0.3$ ). These kinks continue to grow in magnitude and remain localized in the vicinity of the magnetic axis. Eventually, they exceed all other modes in magnitude and the code fails because it can't find a magnetic axis.

Numerical experiments showed that this instability is a subtle one, with its growth rate depending sensitively on the discretization used in the Poisson solver, and not being at all sensitive to numerical details of integrations along field lines and the transformation to magnetic coordinates. This situation is similar to experience with three-dimensional variational codes in flux coordinates, in which quite subtile discretizations and experimentation were needed to obtain numerically stable codes, ${ }^{38}$

Some examples of runs we mede to explore the aumerics of this instability are given in Fig. 12. It mighr be expected that subtracting off $\nabla \times \mathbf{B}$ from Ampere's law and solving for the correction to the new magnetic field $[\mathrm{Eq} .(50)]$, rather than for the field jtself, would improve the accuracy of the code. In particular, this reduces the large numerical cancellation among the terms that make up $B^{p}$, which is tending to zero during convergence. The figure shows that this modification had little effect on the initial convergence rate of the code, and was actually destabilizing for long runs. The figure also shows that switching from the generalized Boozer gauge, Eq. (46), to another gauge, Eq. (49), had little influence on the instability, even though these gauges represent different numerical approximations. In this case, the convergence rate for the two gauges was nearly the same, with the fina! residual slightly smaller for the Boozer gauge. We have found that the gauge of Ey. (49) can be considerably more accurate than the Boozer gauge for axisymmetric equilibria with nearly circular flux surfaces.

The form of the discretization of the Poisson equation, and of the boundary condition for the Poisson equation, was found to play a dominant role in determinug the numerical stability of the code. Changing the radial discretization of the Poisson equation [Eq. (53)] from a nor conservative form to a conservative oue eliminated an instability near the outermost flux surface and greatly reduced the growth rate of the instability observed in Fig. 11.

Further insight into how the initial state evolves towards the final MHD equilibrium is given in Figs. 13 and 14. Figure 13 shows how the vacuum coordinates [of which only two harmonics appear in (a)] turn into the equilibrium maguetic coordinates in (b), which have a more complex spectral represen- 
tation. The Fourier coefficients of $x$ vary slowly and smoothly throughout the interval $0 \leq \rho \leq 1$, which justifies a posteriori the use of uniform radial meshes and of finite differences to approximate derivatives. Differentiating and convolutıng the coordinates gives the metric elements, three of which are shown in Fig. 14. The $J g^{\rho p}$ element has a rather weak dependence on $\rho$, unlike the off-diagonal elements. The magnetic surfaces are noncircular, as indi-

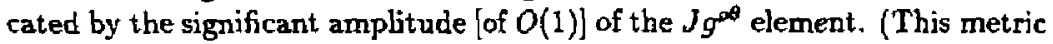
element is not singular near the magnetic axis betause the Jacobian $\mathcal{J}^{p} \propto \rho$ cancels the $1 / \rho$ singularity in $g^{\rho \theta}$ for $\rho \ll 1$.) The weak nonaxisymmetry of the equilibrium solution is apparent in the much smaller magnitude of the $J g^{\rho \phi}$ metric element.

\section{Conclusions}

In this paper, we have discussed a new computer code and have demonstrated its significant potential for advancing the fusion community's ability to investigate three-dimensional MHD equilibria. The code was shown to converge rapidly and efficiently for representatjve tokamak and stellarator equilibria that have magnetic surfaces. It does not assume the existence of magnetic surfaces, an important advance over previous three-dimensional equilibrium solvers. ${ }^{16-18}$ (The self-consistent solution of equilibria with islands and stochastic regions recizires additional numerical machinery, and will be discussed elsewhere.)

The PIES code uses a novel nonvariational algorithm that iterates the MHD equations in special cuordinates. This leads to the successive solution of two linear partial differential equations and nonlinear numerical transformations between laboratory and magnetic coordinates. The hyperbolic magnetic differential equation for the stream function $\nu$ of the current density contains the island physics, while the elliptic Poisson equation derived from Ampere's law contains most of the numerical subtleties that affect overall convergence. This paper studied the simpler case of equilibria with magnetic surfaces, for which extensive intuition has been developed by running two-dimensional and three-dimensional codes.

Our fitst important conclusion is that this algorithm converges. Although the basic algorithm was already considered in the 1950's, our numerical results are the first to show that this algorithm is generally convergent in 
three-dimensions.

Our second important conclusion is that the PIES algorithm not only converges. but converges rather rapidly. Typically of order ten iterations leads to an equilibrium that has the smallest possible residual that is consistent with the parameters ftproc, $M, \mathcal{N}$, and $L$. This is several orders of magnitude fewer iterations than are typically required for variational codes, in which small (but computationally less demanding) steps are taken at each iteration towards an extremum of the variational functional. The rapid convergence shows that there are no vestiges of any fast time scale behavior in the algorithm.

Convergence not only requires few iterations, but each iteration is relatively efficient. Although we made only a modest effort to vectorize the PIES code for running on a CRAY supercomputer and did not use the most efficient algorithms, the code is already competitive with other threedimensional equilibrium codes in that only a few CRAY-XMP minutes are need to attain a stellarator equilibrium to four significant digits. Further improvements could easily be made by using a more efficient algorithm for constructing magnetic coordinates, a more carefully vectorized code for solving block tridiagonal matrices, and optimized coordinates ${ }^{10}$ to reduce the size of the matrix associated with solving Ampère's law. The code also lends itself to parallel computer architectures since the most time consuming part of the code, following $L+1$ field lines to transform quantitites to magnetic coordinates, can be done in parallel and independently on each magnetic surface. Significant speedups should be attainable on forthcoming parallel computers.

A third conclusion is that the PIES algorithm, although convergent, sometimes requires application of a radial filter to remove high-frequency modes (a forrn of radial dealiasing). In the absence of this filtering, highfrequency noise in various Fourier coefficients appears and grows after many iterations. Similar instabilities are well known in meteorological codes, $\stackrel{27}{ }$ and more generally in many strongly nonlinear codes. Our numerical experiments show that the instability is sensitive to the choice of discretization used for Ampère's law and insensitive to many other details of the PIES algorithm, e.g., whether finite differences or splines are used to differentiate the coordinates to get the metric elements, or whether one subtracts off $\nabla \times \mathbf{B}$ from the right side of Ampere's law to remove cancellations between large terms 
[see Eq. (50)], or the choice of gauge in representing the solution to Ampère's law. Numerical experiments show that the instability is related to how well one approximates the correct asymptotic analytic behavior near the magnetic axis; an important element of success was our explicit treatment of leading radial powers when differentiating or averaging Fourier coefficients.

It is our hope that the optimistic conclusions based on the PIES code will stimulate further consideration of nonvariational algorithms and their applications to three-dimensional MHD equilibria with islands and stochastic regions. 


\section{Appendix A. A Simple Analytic Example}

To help the reader understand the PIES algorithm, we give a simple example that can be worked out analytically to lowest order in a small parameter $\epsilon$. We perturb a cylindrical MHD equilibrium by twisting the magnetic field lines a little bit in each magnetic surface (i.e., we perturb the poloidal angle $\theta$ ), and then we use the algorithm to calculate analytically the equilibrium solution.

This example is particularly easy to work out since the magnetic surfaces remain circular at each iteration; only the poloidal angle changes on successive iterations. Two passes through the algorithm recover the unperturbed MHD equilibrium. The first pass has the effect of correcting the initial magnetic field so that its magnetic field lines correspond to an unperturbed $\theta$. The second pass corrects the magnetic coordinates. We sketch the details of the algorithm to $O(\epsilon)$. Carrying out this example to higher order has also been done symbolically using MACSYMA ${ }^{38}$ and proved a valuable aid in debugging the code.

We start with a magnetic field in canonical form [see Eq. (35)]: and assume circular magnetic surfaces and a constant rotational transform:

$$
\psi=\frac{1}{2} b_{b} \rho^{2}, \quad t=t_{0}
$$

This field satisfies the MHD equations, Eqs. (1)-(3), with the following net toroidal current and pressure profiles

$$
\frac{d I}{d \psi}(\rho)=\frac{2 t_{0}}{R}, \quad \frac{d p}{d \rho}=-2\left(\frac{t_{0} b_{0}}{R}\right)^{2} \rho_{1}
$$

where $R$ is the effective Inajor radius of the cylinder (so that $z=R \phi$ ).

We perturb the magnetic field by adding an $l=2$ perturbation to the poloidal angle:

$$
\bar{\theta}=\theta+\epsilon \rho^{2} \sin (2 \theta) .
$$

To $O(\epsilon)$ we can then write the gradients

$$
\begin{aligned}
& \nabla \nu=b_{0} \rho \dot{\rho}, \\
& \nabla \bar{\theta}=[2 \epsilon \rho \sin (2 \bar{\theta})] \dot{\rho}+\frac{1}{\rho}\left(1+2 \epsilon \rho^{2} \cos (2 \bar{\theta})\right) \hat{\theta} \\
& \nabla \phi=\frac{1}{R} \dot{\phi}
\end{aligned}
$$


where $\hat{\rho}, \hat{\theta}$ and $\hat{\phi}$ are the usua] unit vectors of a cylindrical coordinate system defined by $\rho, \theta$ and $z=R \phi$. These gradients directly give the metric elements of the perturbed nonorthogonal coordinate system (e.g., $\left.g^{\bar{\theta} \bar{\theta}}=\nabla \bar{\theta} \cdot \nabla \bar{\theta}\right)$. Substituting the gradients into the canonical form of the magnetic field, Eq. (35), gives a perturbed magnetic field:

$$
\overline{\mathbf{b}}=\nabla \psi \times \nabla \bar{\theta}+t_{0} \cdot \bar{\gamma} \phi \nabla \psi=\left(\frac{t_{0} b_{0}}{R}\right) \rho \hat{\theta}+b_{0}\left(1+2 \epsilon \rho^{2} \cos (2 \bar{\theta})\right) \hat{\phi}
$$

The nonvariational algorithm togins with this perturbed magnetic field, the current and pressure profiles Eq. (A1), a given circular magnetic surface at $\rho=1$, and the parameter

$$
\lambda=\int_{\rho=1} \overline{\mathbf{b}} \cdot d \mathrm{l}=\left(\bar{b}_{\phi}\right)_{0,0}^{k}=R b_{0},
$$

which specifies the net poloidal current through this outermost flux surface (see Eq. (52)).

The first step is to integrate along the field lines of $\overline{\mathbf{b}}$ and to express $\boldsymbol{x}, \boldsymbol{y}$, and $\bar{b}^{\phi}$ in magnetic coordinates. Since we know the relation between $\bar{\theta}$ and $\theta$, Eq. (A2), we can directly obtain these as expansions in 6 . For example,

$$
\begin{aligned}
x & =\rho \cos (\theta) \simeq\left(\rho+\frac{1}{2} \epsilon \rho^{3}\right) \cos (\bar{\theta})-\frac{l}{2} \epsilon \rho^{3} \cos (3 \bar{\theta}) ; \\
\bar{b}^{\phi} & =\overline{\mathrm{b}} \cdot \nabla \phi=\frac{1}{R} \overline{\mathrm{b}} \cdot \bar{\phi} \simeq \frac{b_{0}}{R}\left(1+2 \epsilon \rho^{2} \cos (2 \bar{\theta})\right),
\end{aligned}
$$

with a similar expression for $y$. These expressions immediately give the $\rho$ Jacobian and $\psi$-Jacobian to $O(\epsilon)$. In fact, from Eqs. (29) and (36), we find:

$$
\begin{aligned}
& \mathcal{J}^{\rho}=\frac{\partial \mathbf{x}}{\partial \rho} \times \frac{\partial \mathbf{x}}{\partial \bar{\imath}} \cdot \frac{\partial \mathbf{x}}{\partial \phi} \simeq R \rho\left(1-2 \epsilon \rho^{2} \cos (2 \bar{\theta})\right) \\
& \mathcal{J}^{\psi}=\frac{1}{\bar{b}^{\phi}} \simeq \frac{R}{b_{0}}\left(1-2 \epsilon \rho^{2} \cos (2 \bar{\theta})\right) .
\end{aligned}
$$

The quantity $\mathcal{J}^{\rho} / \mathcal{J}^{\psi}$ is $d \psi / d \rho=b_{\mathrm{o}} \rho$, as was assumed (se - Eq. (37)).

We next work out to $O(\epsilon)$ the field $h$ due to plasma currents. We find 
using Eqs. (40) and (47):

$$
\begin{aligned}
& h_{\theta}=\frac{d p}{d \rho} \sum_{m, n}^{\prime} \frac{J^{\psi}}{n-m} \frac{m}{t m} \sin (n \phi-m \bar{\theta})=L\left(\frac{t_{0} b_{0}}{R}\right) \epsilon \rho^{3} \sin (2 \theta), \\
& h_{\theta}=\int_{0}^{r} I^{\prime} \frac{d \psi}{d \rho} d \rho=\left(\frac{t_{0} b_{0}}{R}\right) f^{2} \\
& h_{\phi}=\int_{\rho}^{1}\left(t_{0}^{\prime} J^{\prime} \frac{d \psi}{d \rho}+\frac{d p}{d \rho} J_{0,0}^{\psi}\right) d \rho=0 .
\end{aligned}
$$

Because $h$ is not divergence free, we have to find a scalar fielo $u(p, \bar{\theta} ; \phi)$ such that $\overline{\bar{b}}=\mathbf{h}+\nabla u+\lambda \nabla \phi$ is solenoidal and such that $\overline{\bar{b}} \cdot \nabla \rho=0$ on the outermost flux surface. From the metric elements, we calculate the right sides of the Poisson equation and of the Neumann boundary condition for the scalar $u$ :

$$
\begin{aligned}
&-\mathcal{J}^{\rho} \nabla \cdot \mathbf{h}=-\left\{\partial_{\rho}\left[\mathcal{J}^{\rho}\left(g^{\rho \rho} h_{\rho}+g^{\rho \bar{\theta}} h_{\theta}\right)\right]+\partial_{\partial}\left[\mathcal{J}^{\rho}\left(g^{\bar{\phi} \rho} h_{\rho}+g^{\bar{\theta} k_{\delta \phi}}\right)\right]\right\} \\
& \simeq-12 t_{0} b_{0} \in \rho^{3} \sin (2 \bar{\theta}) . \\
&-\mathcal{J}^{\rho}(\mathbf{h}+\lambda \nabla \phi) \cdot \nabla \rho=-\mathcal{J}^{\rho} h^{\rho} \simeq-4 \mathrm{t}_{0} b_{0} \in \rho^{4} \sin (2 \bar{\theta}) .
\end{aligned}
$$

Since $u \sim O(\epsilon)$, we only need the operators $\mathcal{J}^{\rho} \nabla^{2}$ and $\mathcal{J}^{\rho} \nabla \rho$. to 0 th order. In this case, these reduce to the operators obtained in the unperturbed cylindrical coordinat system. To $O(E)$, it is then easy to see that the solution to the Poisson equation is [see Eqs. (53) and (54)]

$$
u=-\frac{t_{0} t_{0}}{R} \epsilon \rho^{4} \sin (2 \vec{\theta})
$$

We then find the new magnetic field to $O(\epsilon)$ after one iteration (calculating the covariant form of $\overline{\bar{b}}, h_{i}+\partial_{i} u+\lambda \delta_{i, \phi}$ and rajsing indices with the $g^{i j}$ ):

$$
\overline{\bar{b}}^{p}=0, \quad \bar{\sigma}^{\bar{\phi}}=\frac{t_{0} b_{0}}{R}\left(1+2 \epsilon \rho^{2} \cos (2 \bar{\theta})\right), \quad \overline{\bar{b}}^{\phi}=\frac{b_{0}}{R} .
$$

Force balance is now satisfied to $O(\epsilon)$, but the fields and coordinates afe still not self-consistent; we need to solve for the new magnetic coordinates. The first step, again, is to integrate along the field lines of $\bar{b}$ and to express 2 , $y$, and $\bar{b}^{\phi}$ in the new magnetic coordinates. Since the magnetic surfaces 
remain unchanged, we only have to integrate the field-line equation defining the new angular coordinate:

$$
\frac{d \bar{\theta}}{d \phi}=\frac{\overline{\bar{b}}}{\overline{\bar{b}}}=t_{0}\left(1+2 \epsilon \rho^{2} \cos (2 \bar{\theta})\right) \text {, }
$$

which can be integrated directly to $O(\epsilon)$ :

$$
\bar{\theta} \simeq t_{0} \phi+2 \epsilon \rho^{2} \cos \left(2 t_{0} \phi\right) \text {. }
$$

Substituting this into the expressions for $x$ and $y$ (see Eq. (A5)), gives the Fourier series of $x, y$ along field lines in terms of $(n-t m) \phi$. To $O(\epsilon)$, we see that:

$$
\begin{aligned}
& x=\left(\rho-\frac{1}{2} \epsilon \rho^{3}\right) \cos (\bar{\theta})+\frac{1}{2} \epsilon \rho^{3} \cos (3 \bar{\theta}) \simeq \rho \cos \left(t_{0} \phi\right) \\
& y=\left(\rho-\frac{1}{2} \epsilon \rho^{3}\right) \sin (\bar{\theta})-\frac{1}{2} \epsilon \rho^{3} \cos (3 \vec{\theta}) \simeq \rho \sin \left(t_{0} \phi\right) .
\end{aligned}
$$

This says that the new magnetic angle, $\overline{\bar{\theta}}, i_{\bar{D}}$ identical to the unperturbed cylindrical angle 6 . Thus the new magnetic coordinates are the usual orthogonal cylindrical coordinates. We note that $\mathcal{J}^{\psi}=b_{0} / R$ in the new coordinates so that $\mathcal{J}^{\psi}$, n $=0$, for $m>0$. The new $h$ due to plasma currents is then seen to be:

$$
h_{\rho}=0_{;} \quad h_{\bar{\S}}=\left(\frac{t_{0} b_{0}}{R}\right) \rho^{2} ; \quad h_{\phi}=0 .
$$

This is divergence free, so that the solution to the Poisson equation in the second iteration is $u=0$. Since $\lambda$ remains $R b_{0}$, the new magnetic field, $\mathbf{h}+\nabla \boldsymbol{u}+\lambda \nabla \phi$, is seen to be:

$$
b^{\rho}=g^{\rho \rho} h_{\rho}=0 ; \quad b^{\tilde{\phi}}=g^{\tilde{\theta} \bar{\theta}} h_{\bar{\delta}}=\frac{\epsilon_{0} b_{0}}{R} ; \quad b^{\phi}=g^{\phi \phi}\left(h_{\phi}+\lambda\right)=\frac{b_{0}}{R} .
$$

Thus to $O(\varepsilon)$, we bave obtained a magnetic field that satisfies the MHD equations, consistent with the inposed profiles, Eqs. (AI). 


\section{Appendix B. The Old b Field in the New Magnetic Coordinates}

In generalizing the code to handle magnetic islands with net toroidal current or to use magnetic fields from previous iterations to improve convergence, it is useful to express the Fourier coefficients of a magnetic field in terms of the magnetic coordinates defined by its field lines. In this appendix, we show how this can be done.

At the end of one iteration, the new magnetic field, Eq. (51):

$$
\mathbf{b}=\left(b^{\rho}(\rho, \theta, \phi), b^{\theta}(\rho, \theta, \phi), b^{\phi}(\rho, \theta, \phi)\right),
$$

is known in terms of the magnetic coordinates $(\rho, \theta, \phi)$ of the field from the previous iteration, $\mathbf{B}$. The field lines of $\mathbf{b}$ define a new magneic coordinate system $(\bar{\rho}, \bar{\theta}, \phi)$, and we would like to obtain the Fourier expansion of the quantities

$$
\mathbf{b} \cdot \mathbf{e}_{\bar{\rho}}=b_{\bar{\rho}}(\bar{\rho}, \bar{\theta}, \phi), \quad \mathbf{b} \cdot \mathbf{e}_{\bar{\theta}}=b_{\bar{\theta}}(\bar{\rho}, \bar{\theta}, \phi), \quad \mathbf{b} \cdot \mathbf{e}_{\phi}=b_{\phi}(\bar{\rho}, \bar{\theta}, \phi),
$$

in these new magnetic coordinates.

The first step is to transform Eq. (B1) to cylindrical coordinates $x$ and $y$ :

$$
\begin{aligned}
b^{x}(\rho, \theta, \phi) & =\mathbf{b} \cdot \nabla \mathbf{x}=\mathbf{b} \cdot\left(x_{, \phi} \nabla \rho+x_{, \theta} \nabla \theta+x_{, \phi} \nabla \phi\right) \\
& =b^{\rho} x_{, \rho}+b^{\theta} x_{, \theta}+b^{\phi} x_{, \phi}, \\
b^{\nu}(\rho, \theta, \phi) & =b^{\rho} y_{, \rho}+b^{\theta} y_{, \theta}+b^{\phi} y_{, \phi .}
\end{aligned}
$$

We then follow $b^{\mathbf{x}}$ and $b^{\mathrm{y}}$ along field lines of $\mathbf{b}$, applying the algorithm of Ref. 6. This is done in parallel with the usual scalar fields $\left(x, y\right.$, and $\left.b^{\phi}\right)$, so that the overhead is small and vectorization is achieved. This gives the Fourier expansion of $b^{x}$ and $b^{4}$ in the new magnetic coordinates, $(\bar{\rho}, \bar{\theta}, \phi)$.

Next, we form the covariant components of $b$ in the new magnetic coordinates by appropriate dot products:

$$
\begin{aligned}
b_{\bar{\rho}}(\bar{\rho}, \bar{\theta}, \phi) & =\mathbf{b} \cdot \mathbf{x}_{, \bar{\rho}}=\mathbf{b} \cdot\left(x_{, \bar{\rho}} \overline{\mathbf{x}}+y_{, \bar{y}} \bar{y}\right)=b^{x} x_{, \bar{\rho}}+b^{4} y_{, \bar{\rho}}, \\
b_{\bar{\theta}}(\bar{\rho}, \bar{\theta}, \phi) & =b^{x} x_{, \bar{\theta}}+b^{y} y_{, \bar{\theta}}, \\
b_{\phi}(\bar{\rho}, \bar{\theta}, \phi) & = \begin{cases}b^{x} x_{, \phi}+b^{y} y_{, \phi}+R \mathbf{x} b^{\phi} & \text { finite aspect ratio }, \\
b^{x} x_{, \phi}+b^{y} y_{, \phi}+R^{2} b^{\phi} & \text { infinit : aspect ratio. }\end{cases}
\end{aligned}
$$


Since $b^{\phi}$ is also evaluated along field lines, all quantities on the right side are known Fourier series in the latest magnetic coordinates, $(\bar{\rho}, \bar{\theta}, \phi)$. This gives the required expressions, Eq. (B2).

\section{ACKNOWLEDGMENTS}

We would like to thank A. Boozer and D. Monticello for numerous helpful conversations. We would also like to thank 0 . Buseman for making available to us his Fast Hartley Transform foutines, Chris Kert for discussions of filtering routines used by meteorologists, and John Johnson for a critical reading of an earlier version of this paper. This work was supported by the United States Department of Energy under Contract DE-AC02-76-CHO-3073. 


\section{References}

${ }^{1}$ H. Grad, Pliz.. Fluids 10, 137 (1967).

${ }^{2}$ K. Bol et al., Phys. Rev. Lett. 57, 1891 (1986).

${ }^{3}$ A. H. Reiman and H. S. Greenside, Comput. Phys. Commun. 43, 157 (1986).

${ }^{4}$ A. H. Boozer, Phys. Fluids 27, 2110 (1984).

${ }^{5}$ A. H. Peiman and H. S. Greenside, "Numerical solution of threedimunsional magnetic differential equations," Princeton Plasma Physics Laboratory Report PPPL-2423, February, 1987; submitted to the J. Comp. Physics.

${ }^{6}$ A. H. Boozer, Phys. Fluids 25, 520 (1982).

${ }^{7}$ G. Kuo-Petravic, A. H. Boozer, J. A. Rome, and R. H. Fowler, J. Comput. Phys. 51, 261 (1983).

${ }^{8}$ S. P. Hirshman and H. K. Meier, Phys. Fluids 8, 1985.

${ }^{9} \mathrm{~J}$. Kisslinger and H. Wöbig, Europhysics Conference Aostracts, Vol. 9F, Part I, eds. L. Pocs and A. Montvai (European Physical Society, 1985), p. 453.

${ }^{10}$ A. I. Shestakuv, Comput. Phys. Comm. 31, 227 (1984).

${ }^{11}$ L. M. Degtyarev, V. V. Drozdov, M. I. Mikhailov, V. D. Pustovitov, and V. D. Shafranov, Sov. J. Plasma Phys. 11, 22 (1985).

${ }^{12}$ S. C. Chang and J. J. Adamczyk, J. Comput. Phys. 60, 23 (1985) ; S. C. Chang and J. J. Adamczyk, J. Comput. Phys. 6(', 41 (1985); S. C. Chang, J. Comput. Phys. 67, 91 (1986).

${ }^{13}$ D. A. Monticello, W. Park, R. Izzo, K. McGuire, Comput. Phys. Commun. 43, 57 (1986).

${ }^{14}$ F. Bauer, O. Betancourt, and P. Garabedian, A Computational Method in PJasma Physics (Springer-Verlag, Nev: York, 1982), 2nd edition. 
${ }^{15}$ T. C. Hender, B. A. Carreras, L. Garcia, J. A. Rome, and V. E. Lynch, J. Comput. Pilysics 60, 76 (1985).

${ }^{16}$ S. P. Hirshman and D. K. Lee, Comput. Phys. Commun. 39, 161 (1986).

${ }^{17}$ A. Reiman and A. Boozer, Phys. Fluids 27, 2446 (1984).

${ }^{18}$ L. Spitzer, Phys. Fluids 1, 253 (1958).

${ }^{19} \mathrm{H}$. Grad and H. Rubin in Proc. of the Second United Nations Intemational Conf. on the Peaceful Uses of Atomic Energy (United Nations, Geneva, 1958) 31, p. 190.

${ }^{20}$ J. L. Johnson, H. E. Dahled, J. M. Greene, R. C. Grimm, Y. Y. Hsieh, S. C. Jardin, J. Manickam, M. Okabayashi, R. G. Storer, A. M. M. Todd, D. E. Voss, and K. E. Weimer, J. Comput. Phys. 32, 212 (1979).

${ }^{21}$ A. H. Boozer, Phys. Flujds 26, 1288 (1983).

${ }^{22}$ A. C. Hindmarsh, "Solution of Block-Tridiagonal Systems of Linear Algebraic Equations," Lawrence Livermore National Laboratory, UCID30150, Feb. 1977.

${ }^{23}$ U. Schwenn, Comput. Phys. Comm. 31, 167 (1984).

${ }^{24}$ R. Chodura and A. Schlüter, J. Comput. Phys. 41, 68 (1981).

${ }^{25}$ C. deBoor, A Practical Guide to Splines (Springer-Verlag, New York, 1978).

${ }^{26}$ D. F. Elliott and K. R. Rao, Fast Transforms: Algorithms, Analvses, Applications (Acacdemic Press, New York, 1982).

${ }^{27}$ L. S. Solov'ev and V. D. Shafranov in Review of Plasma Physics, edited by M. A. Leontovich (Consultants Burean, New York, 1970), Vol.V.

${ }^{28}$ M. D. Kruskal and R. M. Kulsrud, Phys. Fluids 1, 265 (1958).

${ }^{29}$ P. Garabedian, Partial Differential Equations (John Wiley and Sons, Inc.: New York, 1964).

${ }^{30}$ D. A. Smith, W. F. Ford, and A. Siai, SIAM Review. ży, 199 (1987). 
${ }^{31}$ R. Shapiro, Rev. Geophys. and Space Phys., 8, 359 (1970).

${ }^{32}$ L. S. Solov'ev, Sov. Phys. JETP 26, 400 (1968).

32J. P. Freiciberg, Rev. Mod. Phys. 54, 801 (1982).

${ }^{34} \mathrm{~S}$. Hirshman, private communication (1987).

${ }^{35} \mathrm{~F}$. Mesinger and A. Arakawa, GARP Publications Series No. 17, Vol. I, August 1976.

${ }^{36}$ MACSYMA Reference Manual, Version Ten, Vols. I and II., The Mathlab Group, Laboratory for Computer Science, MIT, 1983. 


\section{Tables}

TABLE I. Parity under the stellarator symmetry, Eq. (6), and leading radial powers $e_{f}(m)$ for Fourier series representing fields used in the PIES code. All fields lie on the integer mesh, except for $u$ and $\mathcal{J}^{\rho} \nabla \cdot \mathrm{h}$, which lie on the halfinteger mesh.

\begin{tabular}{ccccc}
\hline Field $\mathrm{f}$ & Parity & $e_{f}(0)$ & $e_{f}(1)$ & $e_{f}(m)$ for $m>1$ \\
\hline $\boldsymbol{x}$ & even & 0 & 1 & $m$ \\
$y$ & odd & 0 & 1 & $m$ \\
$\mathcal{J}^{\rho}$ & even & 1 & 2 & $m+1$ \\
$\mathcal{J}^{\psi}$ & even & 0 & 1 & $m$ \\
$\mathcal{J}^{\rho} g^{\rho \rho}$ & even & 1 & 2 & $m-1$ \\
$\mathcal{J}^{\rho} g^{\rho \theta}$ & odd & 2 & 1 & $m-2$ \\
$\mathcal{J}^{\rho} g^{\rho \phi}$ & odd & 2 & 1 & $m$ \\
$\mathcal{J}^{\rho} g^{\theta \theta}$ & even & -1 & 0 & $m-3$ \\
$\mathcal{J}^{\rho} g^{\theta \phi}$ & even & 1 & 0 & $m-1$ \\
$\mathcal{J}^{\rho} g^{\phi \phi}$ & even & 1 & 2 & $m+1$ \\
$b_{\rho}$ & odd & 1 & 0 & $m-1$ \\
$b_{\theta}$ & even & 2 & 1 & $n$ \\
$b_{\phi}$ & even & 0 & 1 & $n$ \\
$\mathcal{J}^{\rho} \nabla \cdot \mathrm{h}$ & odd & 1 & 2 & $m+1$ \\
$u$ & odd & 0 & 1 & $m$ \\
$b^{\rho}$ & odd & 1 & 0 & $m-1$ \\
$b^{\theta}$ & even & 0 & -1 & $m-2$ \\
$b^{\phi}$ & even & 0 & 1 & $m$ \\
\hline \hline
\end{tabular}


TABLE II. Physics data needed as input to the PIES code. Either $I^{\prime}$ or $g^{\prime}$ is specified, with the other profile being determined by Eq. (40). The magnetic axis and outermost magnetic surface are determined by field line integrations, as described in Ref. 6. The locations of the axis and boundary are the only information retained from $\mathbf{B}$.

\begin{tabular}{cc}
\hline \hline Array & Definition \\
\hline$R$ & major radius of domain \\
$N$ & number of periods in stellarator \\
$B^{x}(x, y, z)$ & $x$ component of initial $\mathbf{B}$ field in lab coordinates \\
$B^{y}(x, y, z)$ & $y$ component of initial $\mathbf{B}$ field in lab coordinates \\
$B^{x}(x, y, z)$ & $z$ component of initial $\mathbf{B}$ field in lab coordinates \\
$d p / d \rho$ & derivative of pressure profile \\
$d J / d \rho$ & derivative of net toroidal current profile \\
$d g / d \rho$ & derivative of net poloidal current profile \\
$\lambda$ & total poloidal current on outermost magnetic surface \\
\hline
\end{tabular}

TABLE IIl. Numerical parameters needed for the PIES code. These are the most important of the many parameters set in the code. See Ref. 6 and Ref. 7 for more details, and a discussion of these other parameters.

\begin{tabular}{cc}
\hline Parameter & Definition \\
\hline$M$ & maximum poloidal mode number \\
$\mathcal{N}$ & $L+1$ is number of magnetic surfaces \\
$L$ & maximum toroidal mode number \\
$\overline{\mathbf{m}}$ & true for infinite aspect ratio, false otherwise \\
cyl & accuracy of Fourier coeffs from field line following \\
ftproc & convergence precision for MHD equations \\
convg & effective machine precision $(|x|<$ rndoff $\Rightarrow x=0)$ \\
rmdoff & blending parameter for $B^{\phi}(\rho, \theta, \phi)$ \\
bblnd & blending parameter for $x(\rho, \theta, \phi)$, and $y(\rho, \theta, \phi)$ \\
xyblnd & biending parameter for $t(\rho)$ \\
iblnd & diffusion smoothing element in Eq. $(60)$ \\
s1 & antidiffusion smoothing element in Eq. $(60)$ \\
s2 &
\end{tabular}




\section{Figures}

FIG. 1. Schematic relation between the Cartesian coordinatc.s $(X, Y, Z)$, the laboratory coordinates $(x, y, z)$, and the magnetic coordinates $(p, \theta, \phi)$. $\{x, y, z)$ forms a right-handed orthogonal coordinate system, while $(\rho, \theta, \phi)$ is right-handed but generally not orthogonal. $R$ is the major radius.

FIG. 2. Residual versus radial resolution $L$ on a log-log plot. The residual is calculated after one iteration of the PIES code, starting with the analytic equilibrium [Eq. (64)]. The straight line was obtained by a least squares fit, giving an approximate quadratic dependence residual $\simeq 0.1 L^{-1.9}$ of residual on radial resolution $L$.

FIG. 3. $(\rho, \theta)$ grid for the 0th (Part a) and 16th (Part b) iterations of the perturbed Solov'ev equilibrium, Eq. (67). There is a Shafranov shift of about $10 \%$. The final state, (b), closely approximates the analytic Solov'ev solution.

FIG. 4. Residuals [Egs. (61)] versus iteration number $K$ for the perturbed Solov'ev equilibrium for $M=10, \mathcal{N}=0, L=20$, and ftprec $=10^{-5}$. In (a) no filtering is used ( $s 1=s 2=0)$, while in (b) $s 1=1 / 2$ and $s 2=$ $-1 / 2$. Residuals are given when no fields are blended (---), when the rotational transform only is blended (-i-), when the coordinate $x$ is blended $(-x)$, and when all three fields are blended (bix). The blending parameter is $1 / 2$ in all cases. Curves that stop before the 17 th iteration diverged because of numerical instabilities.

FIG. 5. Plot of residual versus radial resolution $L$ for a fixed iteration number of twenty iterations and for several different values of the Fourier precision parameter ftproc. The latter is seen to provide a cutoff to the residual, which can not get smaller than about this amount. For the smallest values of $f t p r e c$, the residual is converging quadratically with approximately the same constant and exponent as given in Fig. 2, until the ctitoff of ftproc is reached.

FIG. 6. Plot of the Fourier coefficients of the cylindrical coordinate $x$ versus t.1e radial variable $o$, for the perturbed Solov'ev axisymmetric equilibri im. Part (a) shows the Fourier coefficient for the initial iteration, Part (b) shows the Fourier coefficients of the converged solution. 
FIG. 7. Initial (a) and final (b) plots of the Fourier coefficients of $B^{\rho} / B^{\phi}$ versus $\rho$ for he perturbed Solov'ev equilibrium.

FIG. 8. The $(\rho, \theta)$ grid in the $\phi=0$ plane for the initial (a) and final (b) states of the stellarator field. $L=20, M=6, \mathcal{N}=3$, ftprec $=10^{-5}$.

FIG. 9. Residual versus jteration number $K$ for the low-shear steilarator equilibrium. In (a) no filtering is used ( $s 1=s 2=0)$, while in (b) $s 1=1 / 2$ and $s 2=-1 / 2$. The blending parameter is $1 / 2$ in all cases.

FIG. 10. Fesidual versus iteration number $K$ for the low-shear stellarator equilbrium, for no blending and different choices of filtering parameters $s 1$ and $s 2$ isee Eqs. ( 59 ) and (60)!. The curves are labeled by ordered pairs $(s 1,-s 2)$.

FIG. 11. Fourjer coefficients of $B^{p} / B^{\phi}$ as functions of $\rho$ during successive iterations of the PIES code in the absence of radial filtering. A slowly growjng instability near the magnetic axis eventually dominates the Fourier modes, preventing convergence.

FIG. 12. Residual versus iteration number $K$ for different chnices of the flags rwerlf and brrggf. The former flag if true subtracts of $\nabla \times B$ when solving Ampère's law as in Eq. (50); if falso, no subtraction is performed. Simjlarly, if barggf is true, the expression Eq. (47) is used to evaluate $h$ in terms of $\mathrm{J}$, otherwise Eq. (49) is used.

FIG. 13. Fourier coefifients of the cylindrical coordinate $x$ for the stellarator field.

FIG. 14. Fourier coefficients for three of the six metric elements of the converged stellarator equilibrium. 


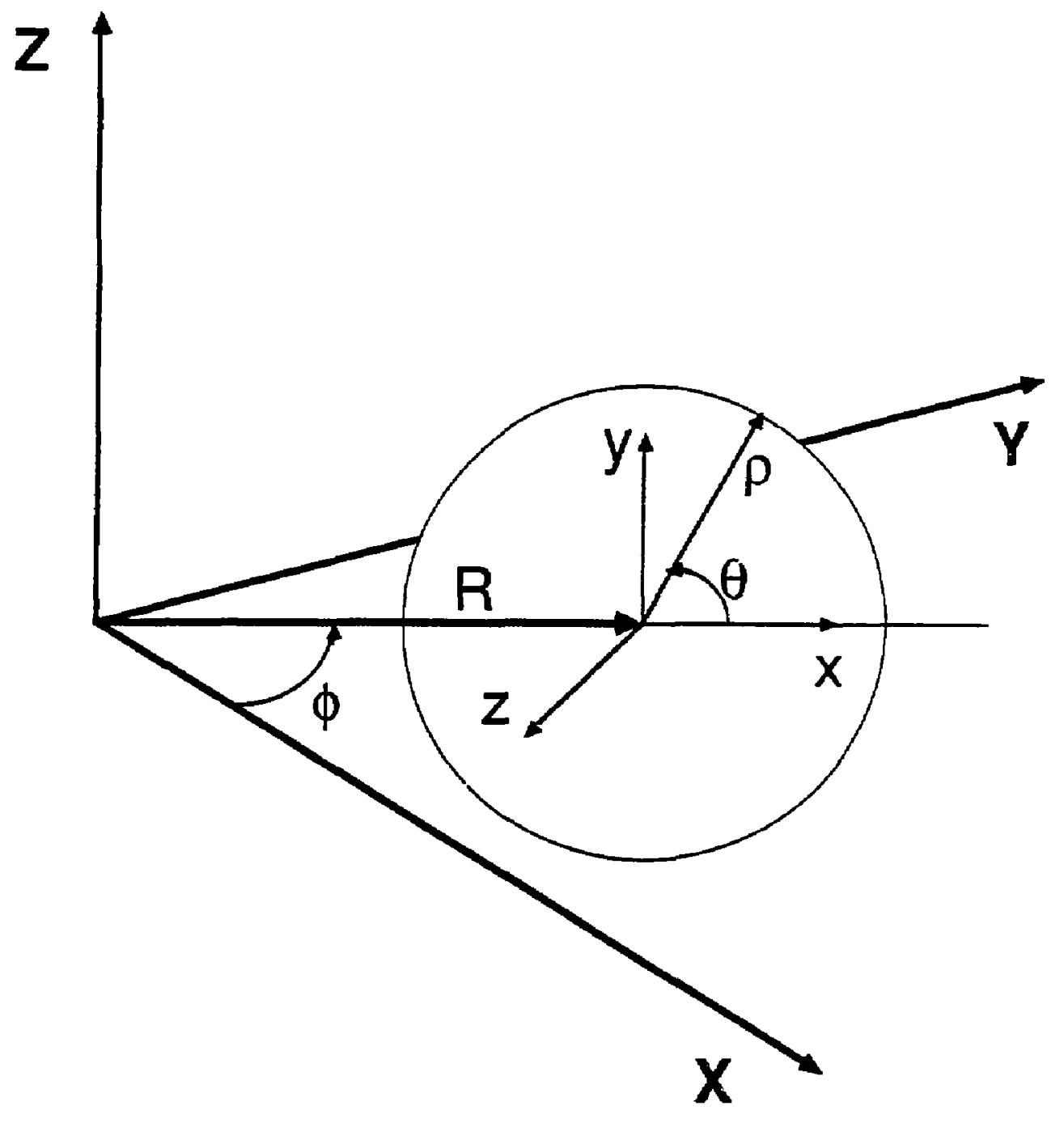

Fig. 1 


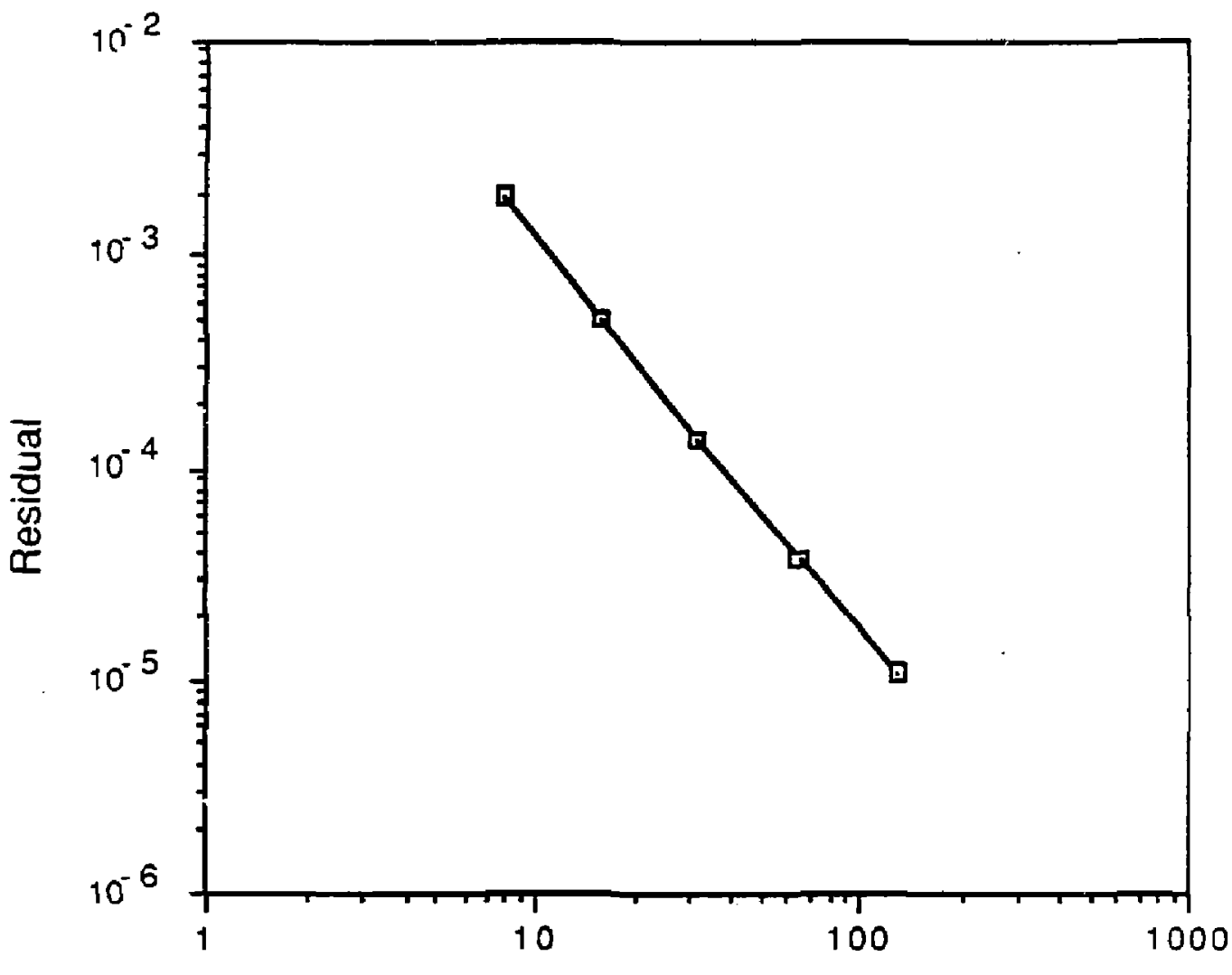

Radial Resolution L

Fig. 2 

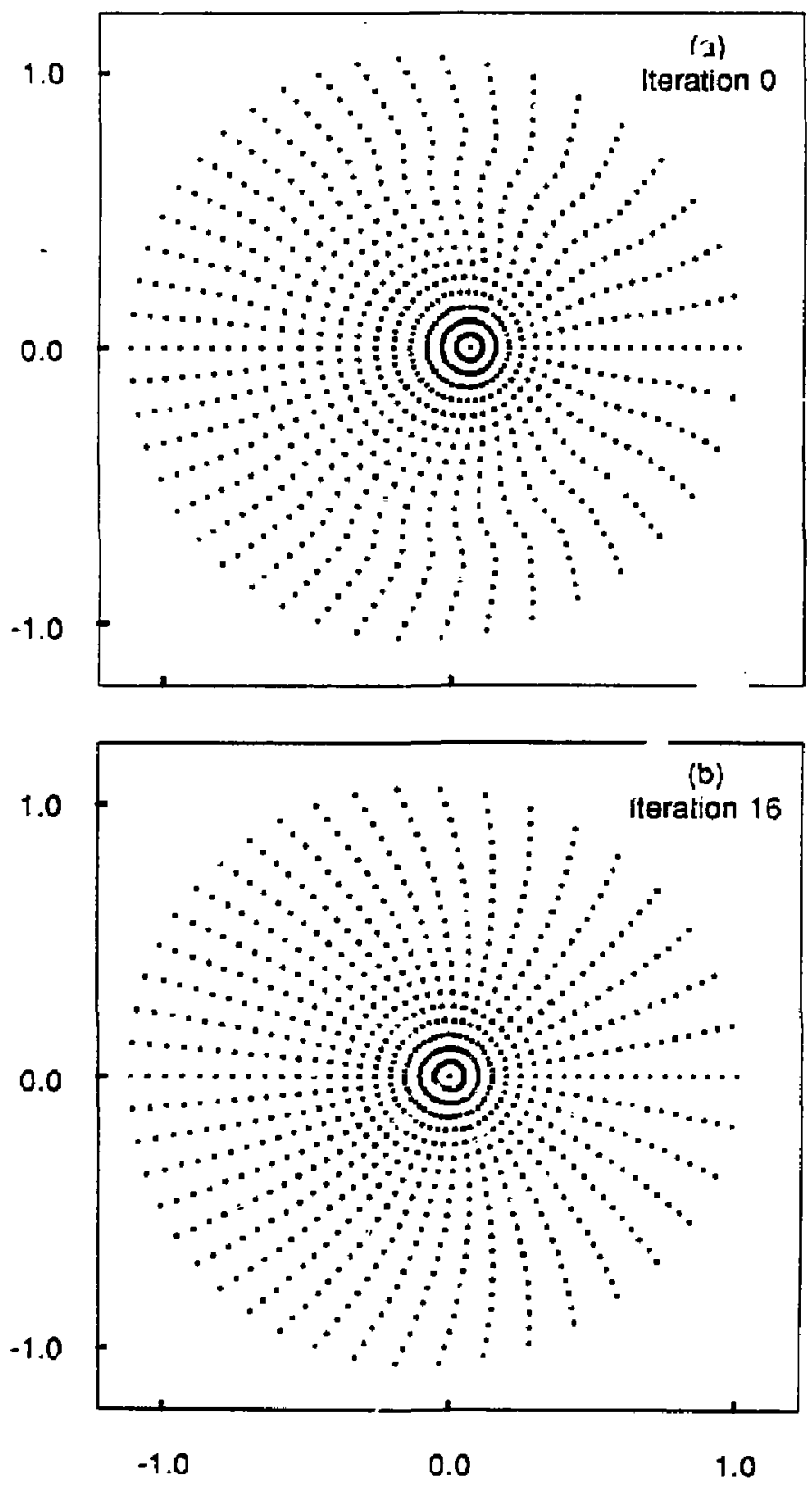

Fig. 3 

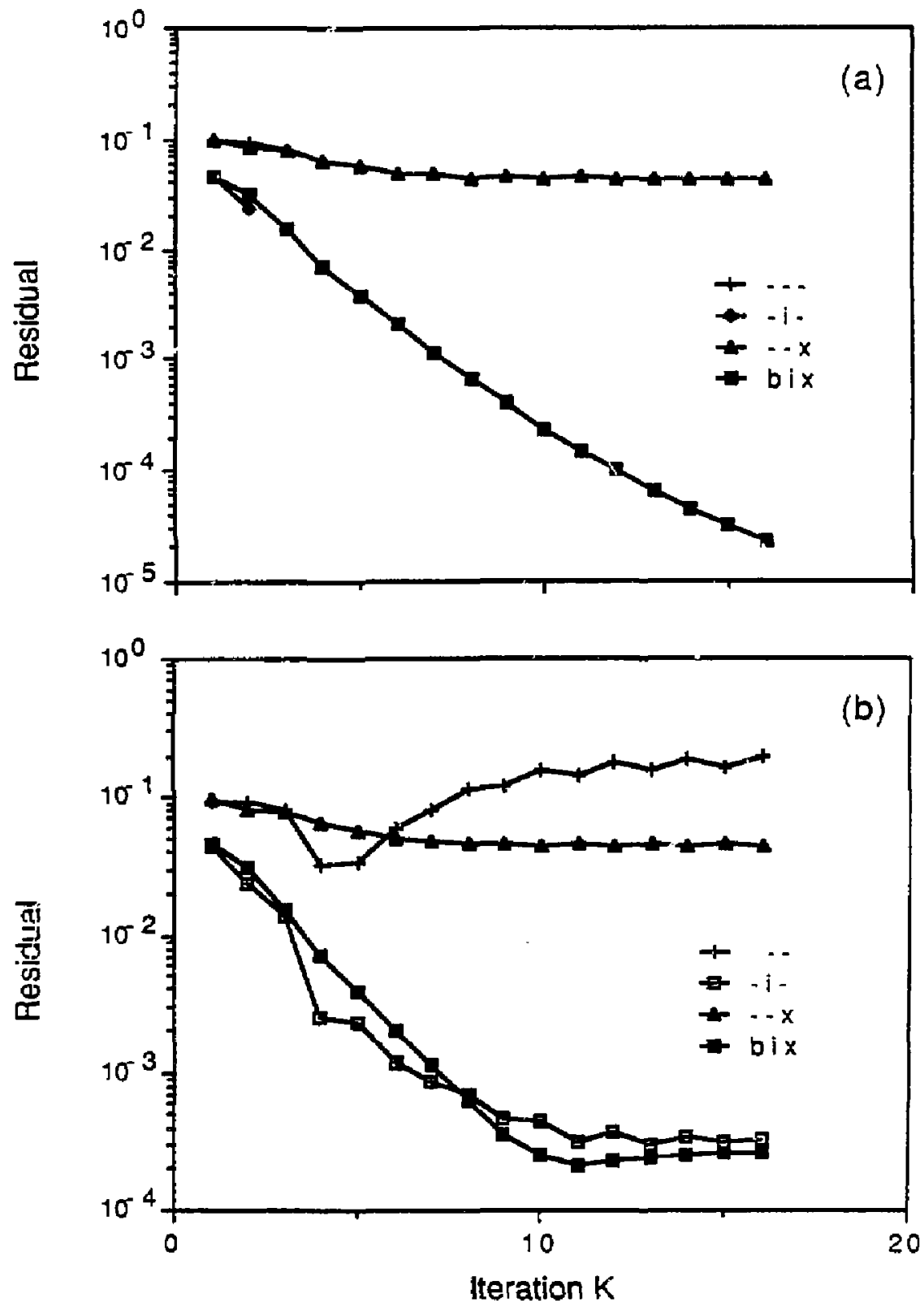

Eig. 4 


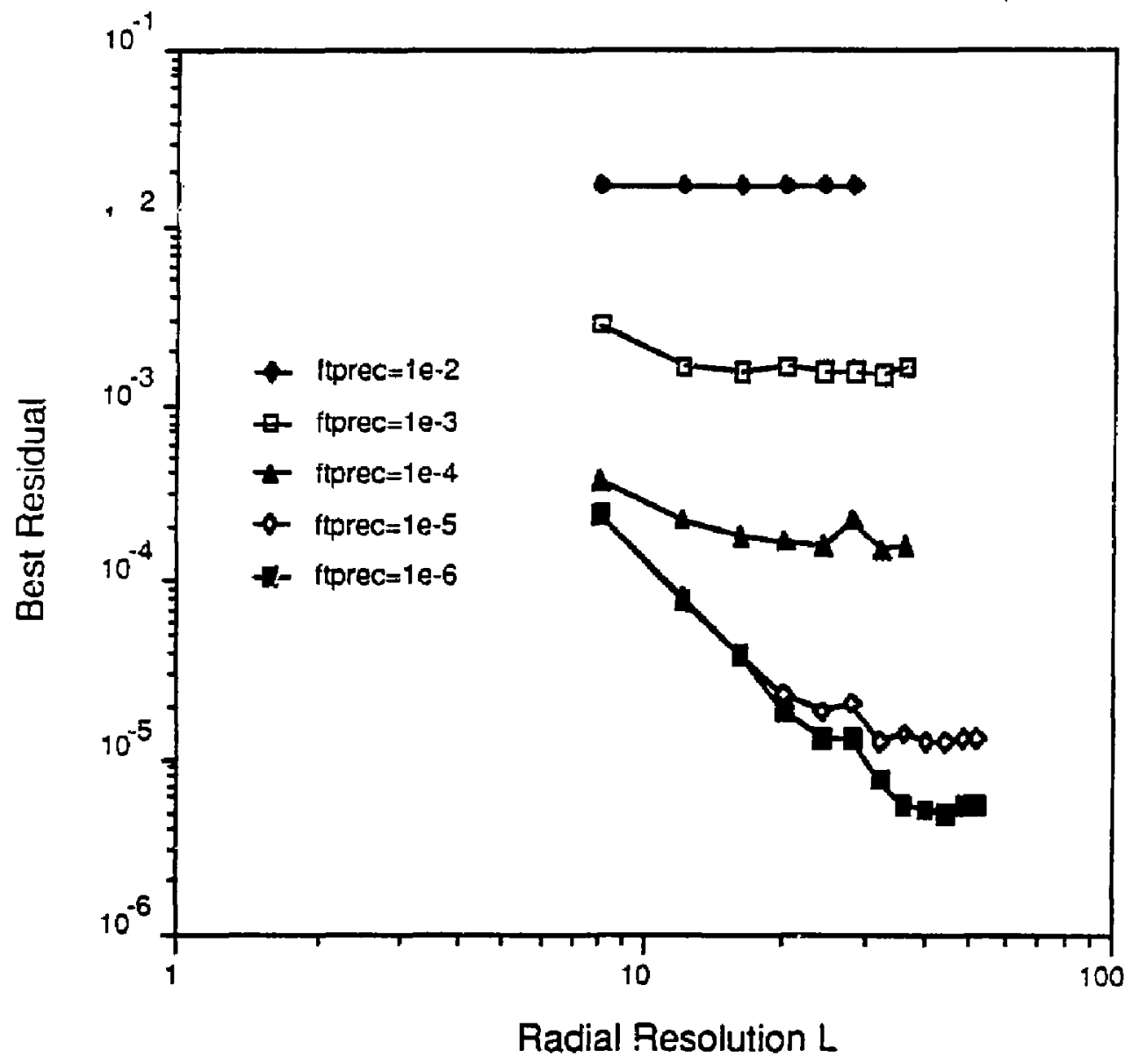

Eig. 5 

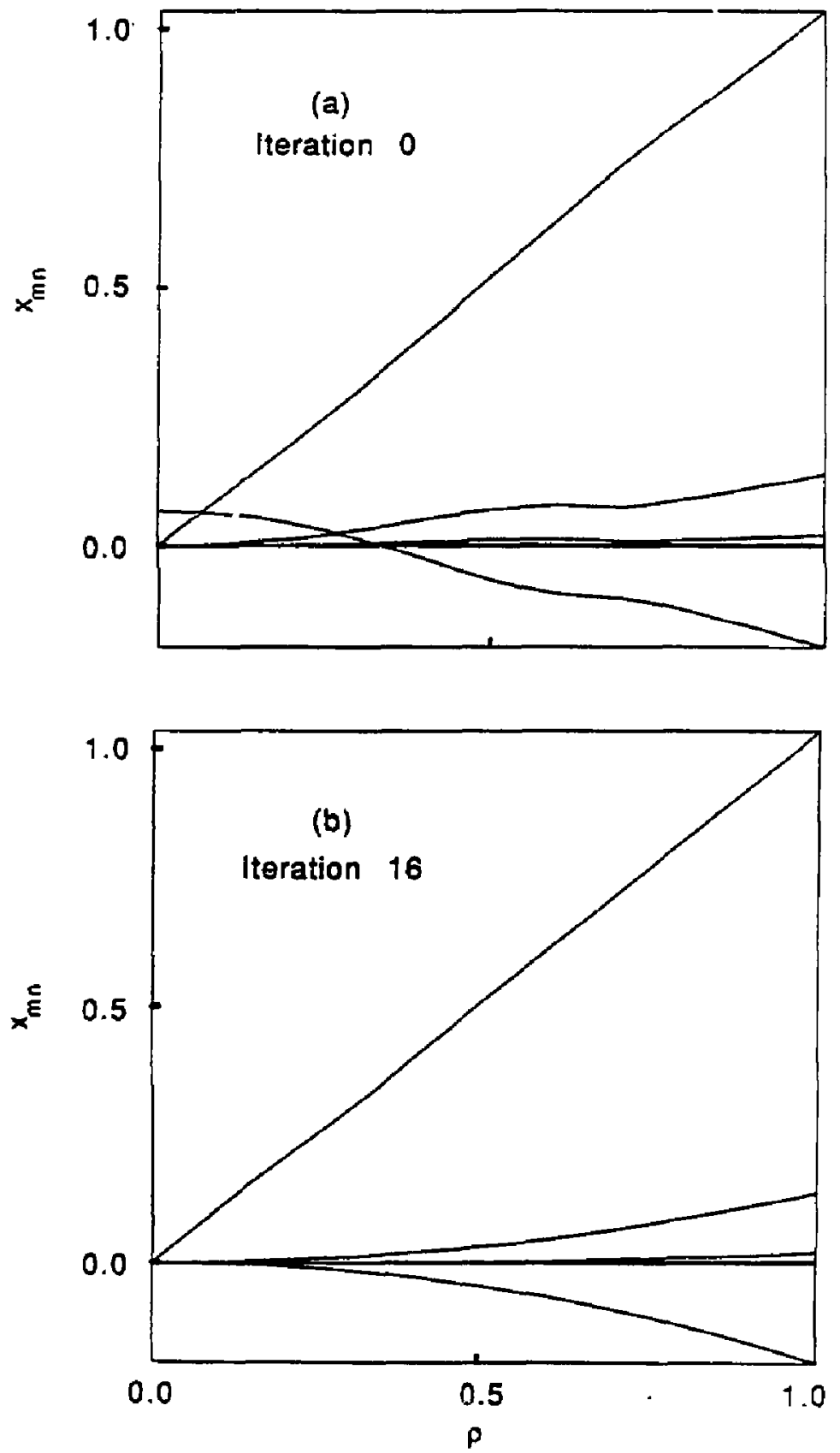

Fig. 6 

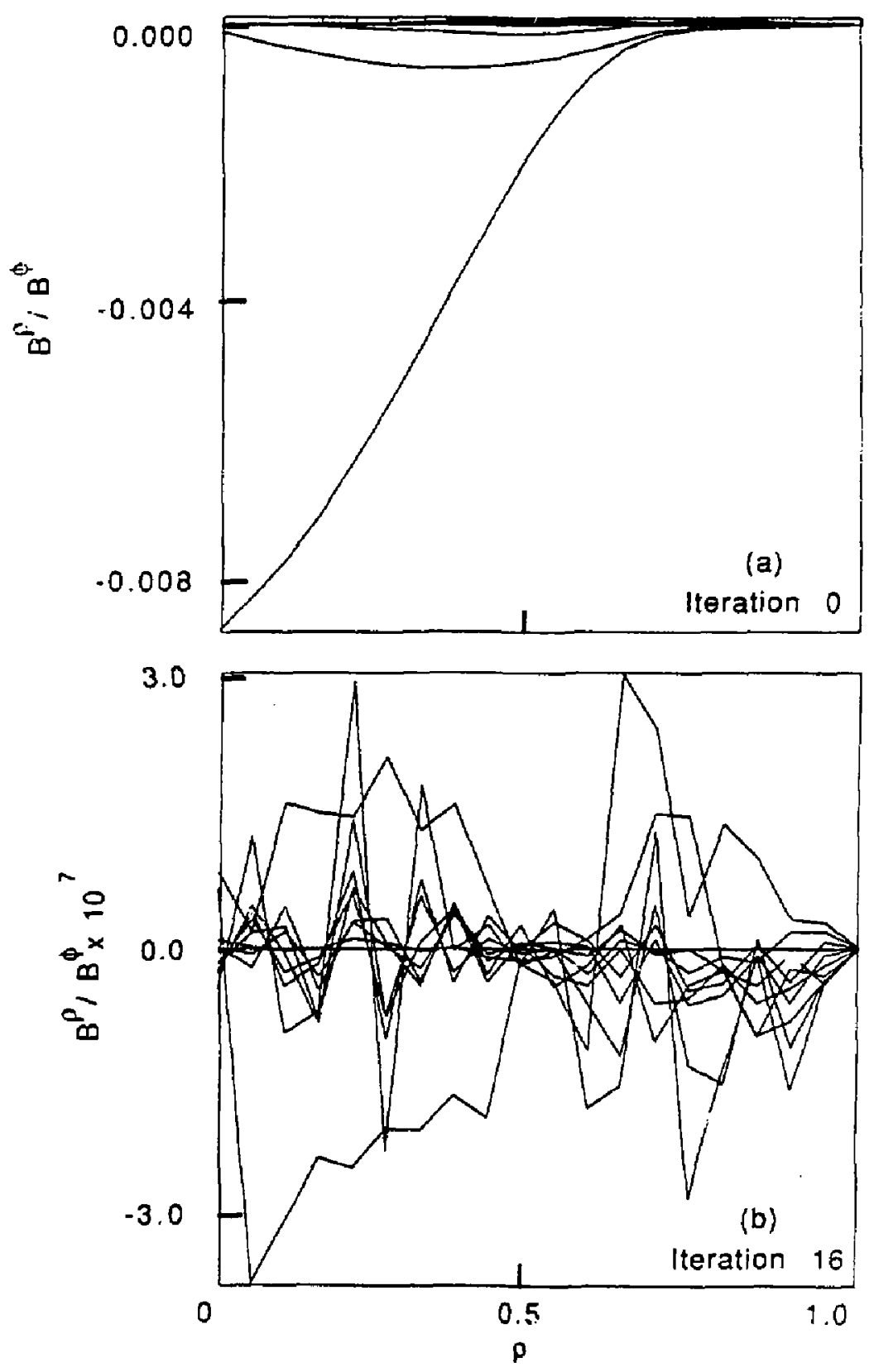

Fig. 7 

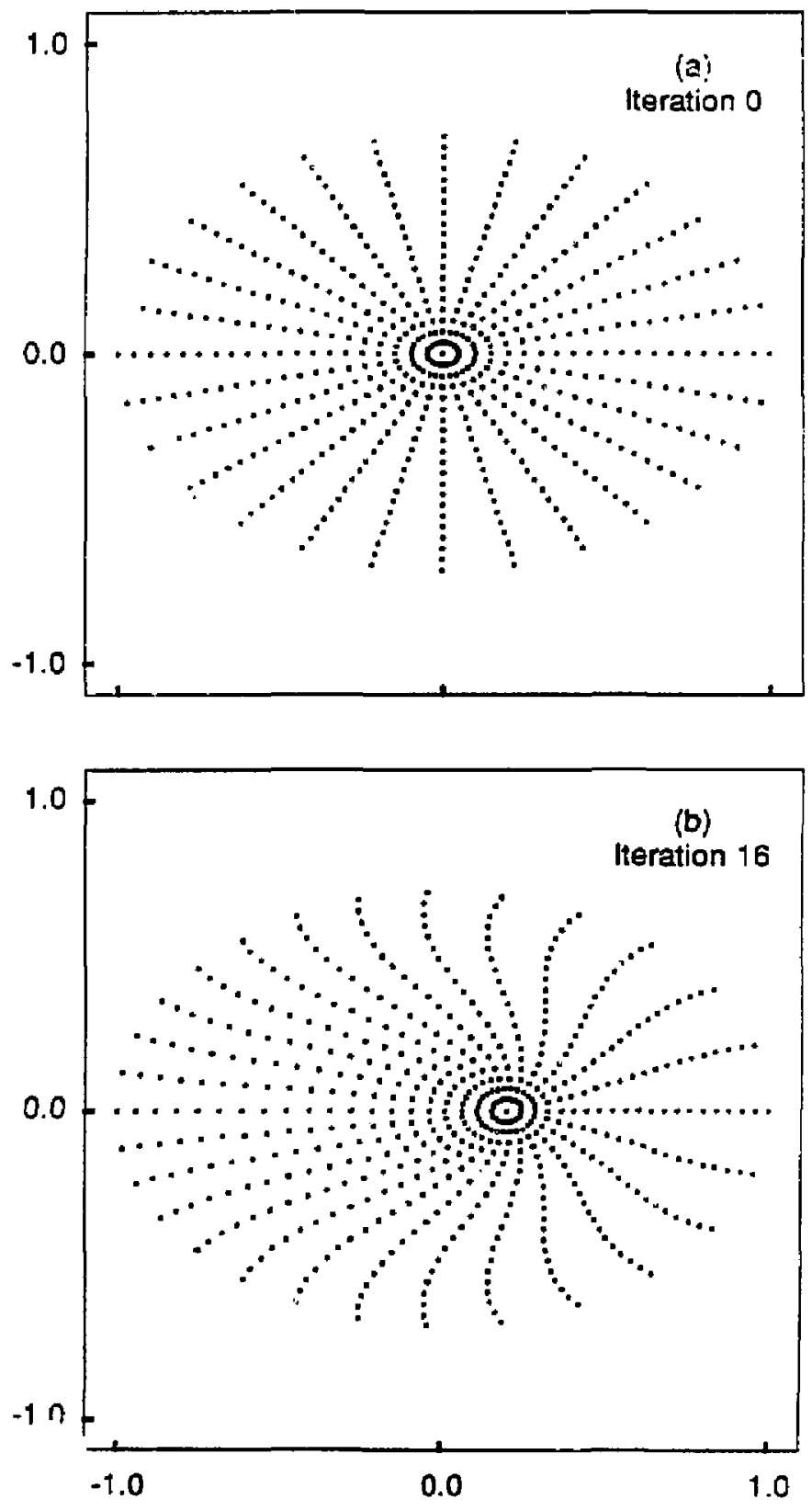

Fig. 8 

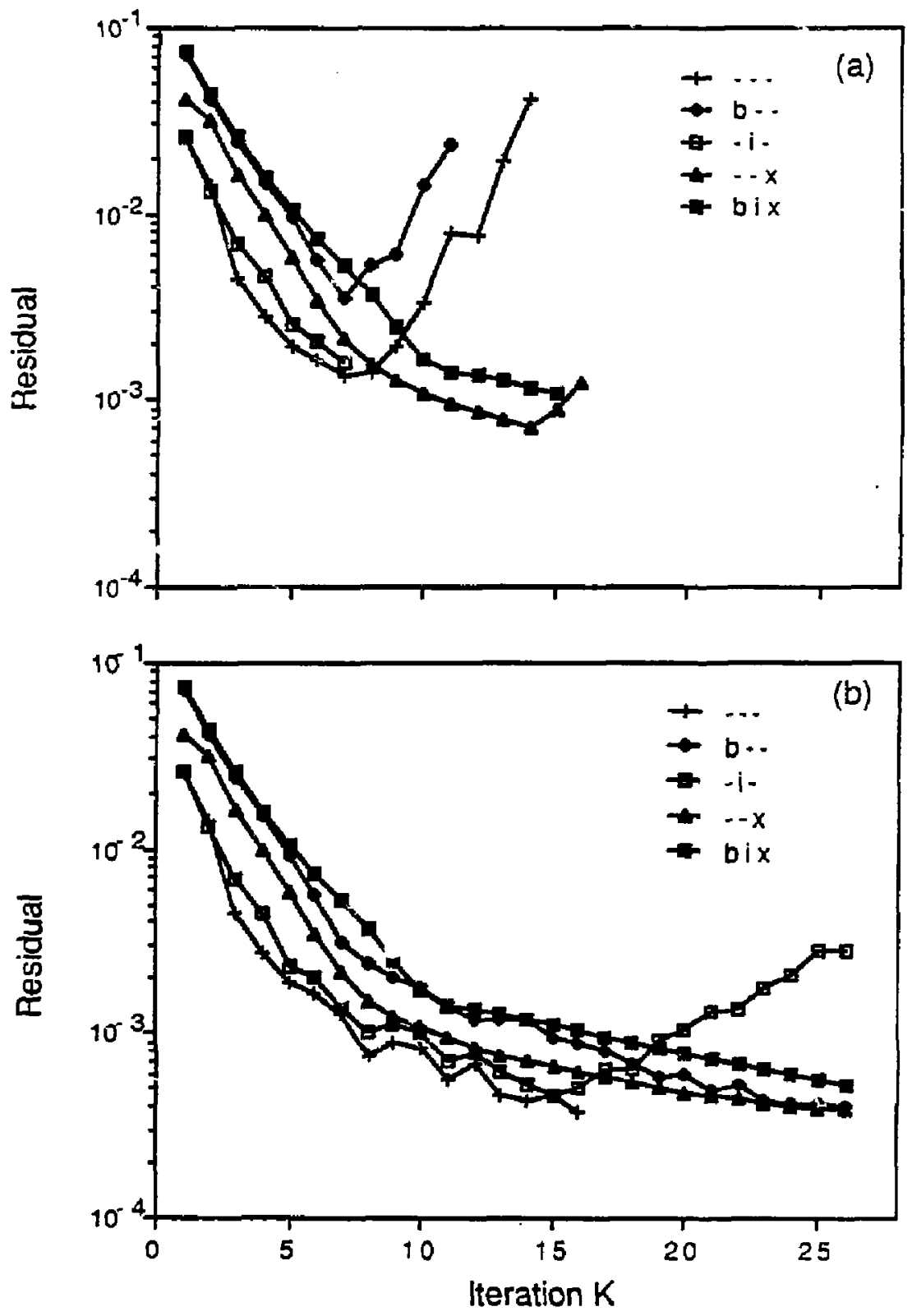

Fig. 9 


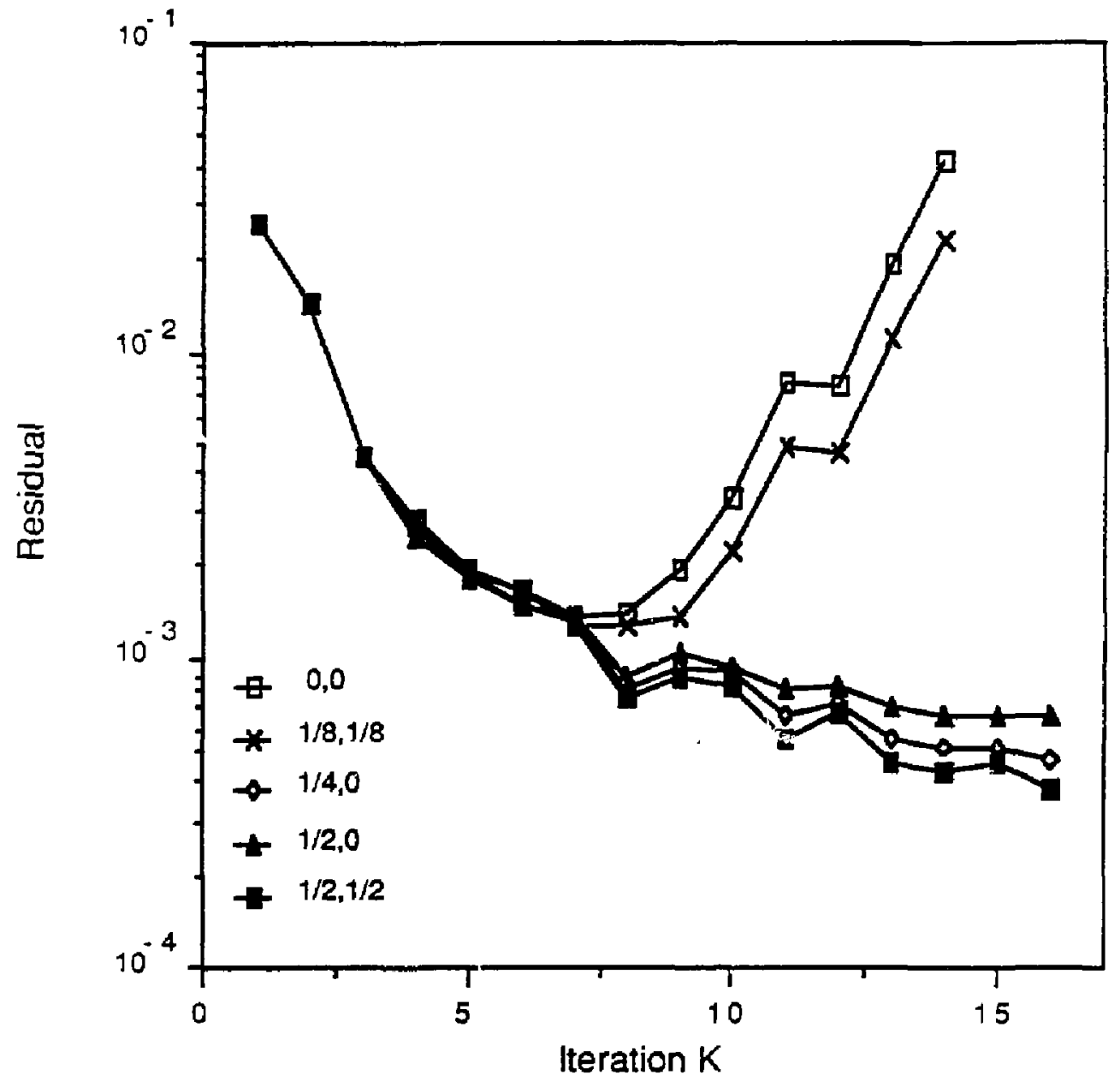

Fig. 10 

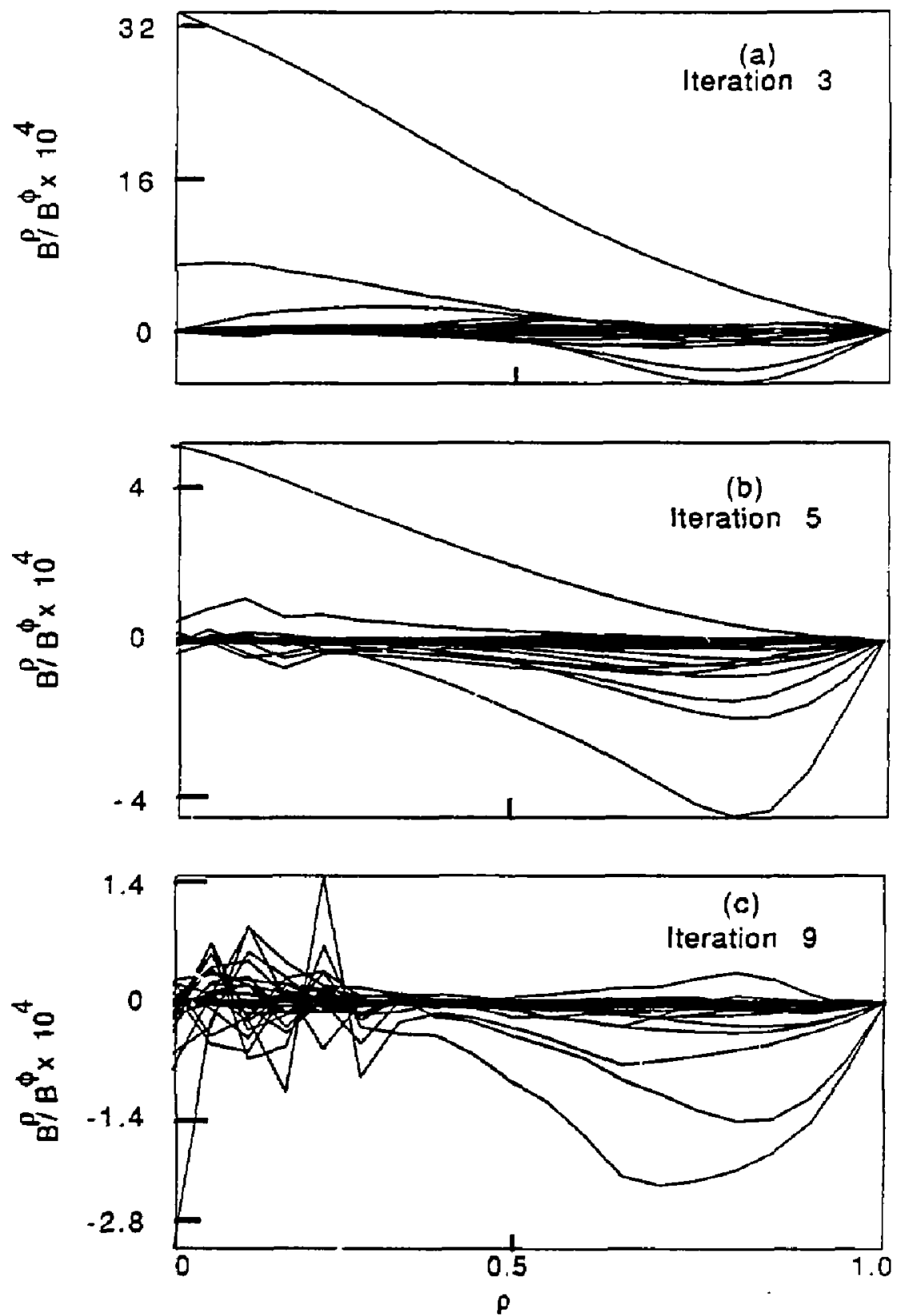

Fig. 11 


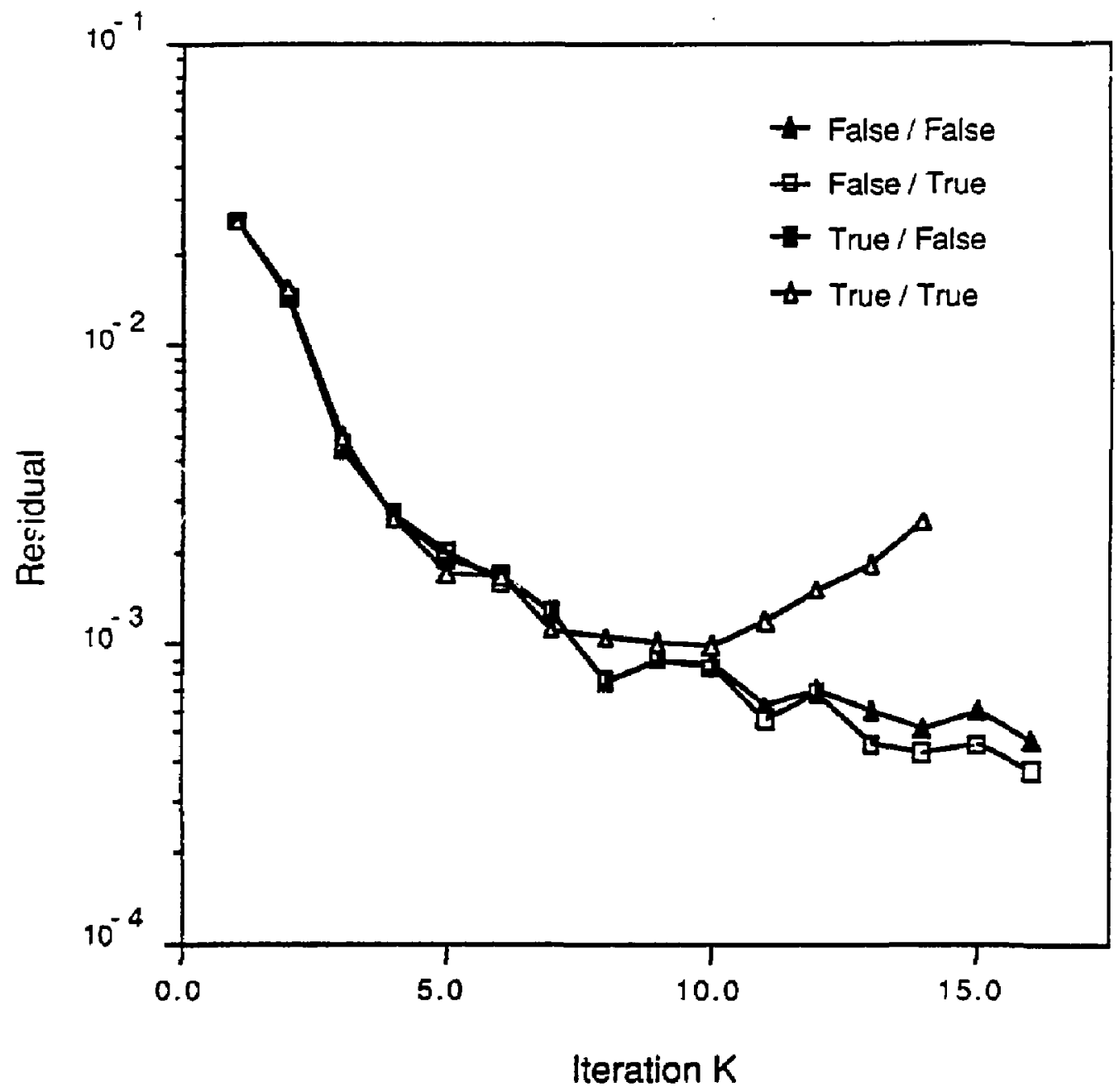

Fig. 12 

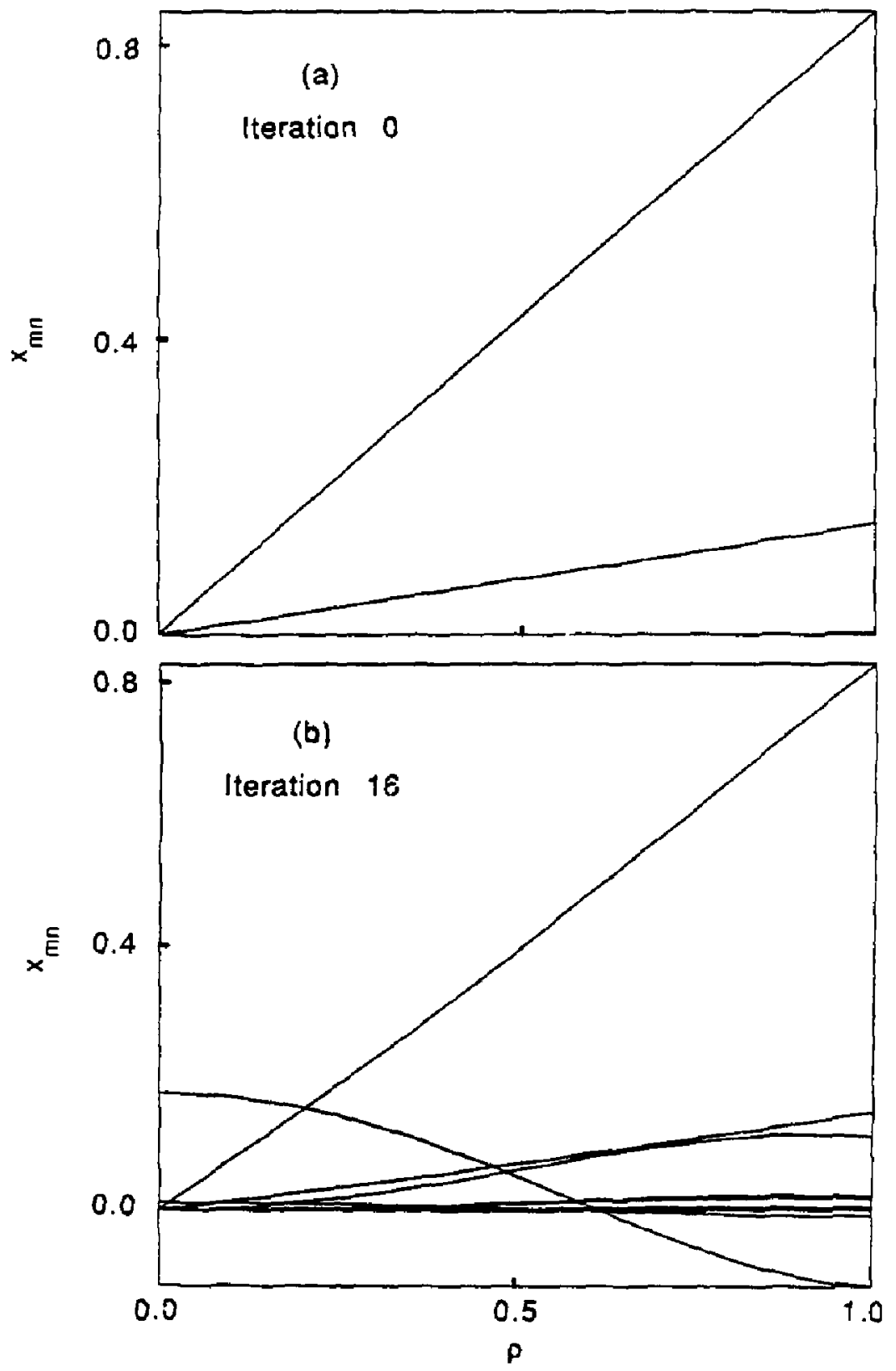

Fig. 13 

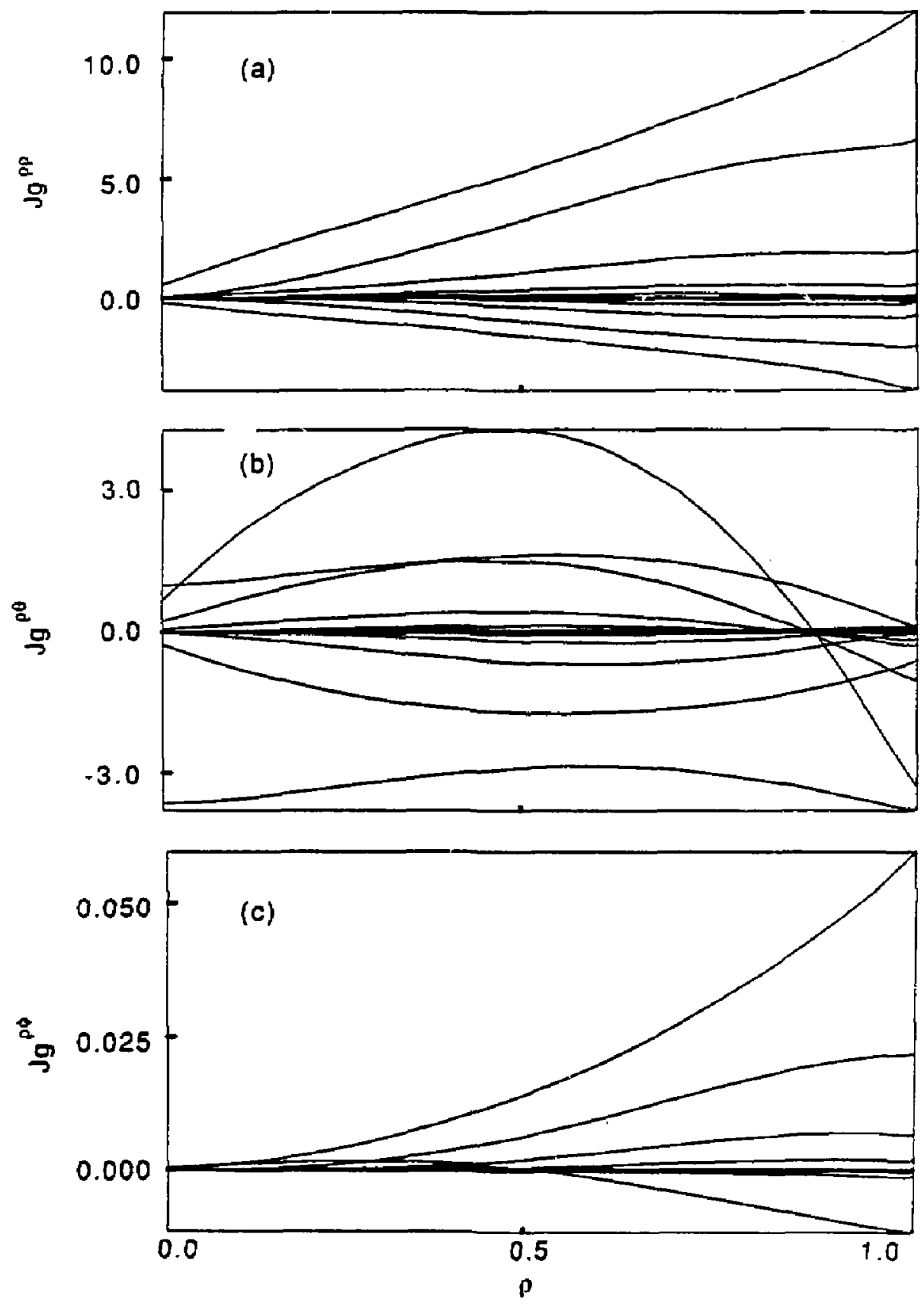

Fig, 14 
Dr. Frank J. Paolonl, Unlu ot wollongong, AUSTRALIA

Prof. M.H. Erannan, Unly Sydnoy, AUSTRALIA

Plasma Research Lab., Australlan Mat. Univ., AuSTRALy

Prof. I.R. Jones, FIInders Unlv., AUSTRRLIA

Prof, f. Cap, Inst Theo Phys AUSTRIA

Prof. W. Helndier, Institut fur Theoretische Physik,AUSTRIA

M. Goossens, Astronomisen InstituUt, BELGIUPA

Ecole Royale Mllitaire, Lab de Phys Plasmas, BeLGILA

Con, of Eurogan, Dg XII fusion Prog, BELGIUH

Prot. R, Bowcique, Loboratorlon voor Natuarkunde, BELGIU:

Dr. P.H. Sakmnake, Jalv Estadual, ERazil

Inst l tuto De Pesquisas Espaclasi-IRPE, ERril L

Llbrary, Atomlc Energy of Ganado LImIted, CWWDA

Dr. M.P. Bechynskl, IPB Technologles, InC., CANADA

Dr. H.M. Skarsgard, Unlv of Saskatcheman, CNADA

Or. H. Earnard, University at British Coluabia, CANADA

Prof. J. Telchann, Univ. of Montreal, CAMADA

Prof. S.R. Sreanivasan, Univarsity of CeIgary, CANAOA

Prof. Tudor Wo Johnston, IMRS-Energle, CAMAOA

Or. Car. James, Univ, of Albarta, CAwide

Or. Poter Lukec, Komenskeho Univ, CzECHOSLOVAXIA

The LIbrarlan, Culhen Laboratory. ENGLAND

Mrs. S.A. Hutehinson, JET Library, ENGLAO

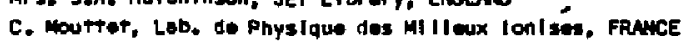

J, Radot, CEN/CNDAANCHE - Bat \$06, FRAnCE

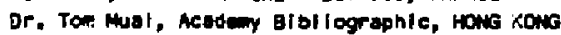

Preprint Library, Cont Rea Inst Phys, Hukgary

Dr. B. Dasgupte, Sahe Inst, IloIA

Or. R.K. Chhejlanl, VIkre Univ. IlolA

Or. P. Kew, Instltute for Plese Researeh, IRDIA

Dr. PhIlllp Rosenuw, Israel Inst Tweh, I SRMEL

Prot. S. Cupersan. Tol Aviv UnIversity, ISRAEL

Librarlan, Intil Cor Theo Phys, ITALY

Prof, G. Rostegn 1, Un1Y D1 Pedove, ITHLY

MIge Clelle de Palo, Aseoc ElRATOA-ENEA, ITALY

albjloteca, del ONR EUATOM. ITAYY

Dr. H. Yemato, Toshlibe Res a Dev, JMPAM

Prof. I. Kerrakeal, Atoalc Enorgy Rese, Instltute, JAPAN

Prof. Kyojl Nisnlkera, Unlv of HIrothims, JAPN

DI rec. Dept. Lo. Tokanak Res. JAERI, JAPAN

Prot. Sutouhl I toh, Kyushu University, JAPN

Research into Conter, Negoya University, JAPN

Prof. S. Tenswe, Kyoto Universlity, JAPM

Llbrory, Kyoto UnIversity, JAPAY

Prof. Nobuyuki Inowe, University of TOKYO, JAPAH

S. Hor I, JAERI, JAPAH

MH. KIm, Koras Advanesd Enargy Rosureh Institute, KOREA

Prof. D.l. Chal, Adv, Inst Sel \& Teen, KOREA

Prof. B.S. LIIE, UnIvarslty of Wolkato, NEW ZEALAO

Instltute of Plasen Physles, PEOPLE's Repuelic of OHINA

LJbrarlen, institute of Phys.. PEOPLE'S REPULlC OF CHINA

Library, Tsing Hua Unlversity, PEOPLE'S REUUBLIC OF CHIMA
Z. LI, Southwest inst. Physlas, PEOPLE'S REPU⿴LIC OF CHIMA Prof. J.A.C. Cabral, Inst Superior Jecn, PORTUGAL

Dr. Octovian Petrus, AL I CUZA Univarsity, ROMANIA Dr. Johan de viljlers, Plasm Physles, AEC, SO AFRicA

Prof. M.A. Helliberg, Unlyersity of Natal, SO AFRICA

Fusion OIV. LIbrary, JEN, SPAIN

Or. Lonnart stonf10, UnIversity of UMEA, SWEDEN

LIbrary, Roval Inst Teeh, SWEEEN

Prof. Hans WI thelmon, Chalmara UnIY TOCin, SWEDEN

Contro Phys dod Plasmos, Ecole Polytech Fed, SwItzealind Bibl lotheek, Fom-l nst Voor Plasma-Fyslca, ThE NETHERLANOS Dr. D.D. Ryutov, SIberlan Aced Scl, USSQ

Dr. G.A. Eliseev, Kurchatav Institute, USSR

Dr. V.A. Glukhlkh, Inst Electro-Physleal, USSR

Dr. V,T, Tolck, Inst, Phys, Tach, USSR

Dr, L.M, Kovrlzhnykh, Institute Gen, Physlcs, USSR

Prol. T.J.M. Boyd, Univ College $N$ Wales, WhLES

Huelear Res. Establlshment, Jullich Łtd., W. GERMaNY Blbllothek, Inst. Fur Plasmaforschung, W, GERMANY Dr. K. Senindter, Ruhr Untvorsltat, W. GERMay ASOEX Reading Re, IPP/Max-Planck-I nst I tut fur

Plasmaphyslk, W. GeFaAry

LIbrarIan, Max-PIanck Institut, W. GERMWY

Prot. R.K. Janev, Inst Phys, Yugoslavia 